\title{
Modeling the Effect of Polymer Chain Stiffness on the Behavior of Polymer Nanocomposites
}

DOI:

10.1021/acs.jpcb.7b02502

\section{Document Version}

Accepted author manuscript

Link to publication record in Manchester Research Explorer

\section{Citation for published version (APA):}

Burgos Marmol, J. J., Alvarez-Machancoses, O., \& Patti, A. (2017). Modeling the Effect of Polymer Chain Stiffness on the Behavior of Polymer Nanocomposites. The Journal of Physical Chemistry Part $B$.

https://doi.org/10.1021/acs.jpcb.7b02502

\section{Published in:}

The Journal of Physical Chemistry Part B

\section{Citing this paper}

Please note that where the full-text provided on Manchester Research Explorer is the Author Accepted Manuscript or Proof version this may differ from the final Published version. If citing, it is advised that you check and use the publisher's definitive version.

\section{General rights}

Copyright and moral rights for the publications made accessible in the Research Explorer are retained by the authors and/or other copyright owners and it is a condition of accessing publications that users recognise and abide by the legal requirements associated with these rights.

\section{Takedown policy}

If you believe that this document breaches copyright please refer to the University of Manchester's Takedown Procedures [http://man.ac.uk/04Y6Bo] or contact uml.scholarlycommunications@manchester.ac.uk providing relevant details, so we can investigate your claim.

\section{OPEN ACCESS}




\author{
Modeling the Effect of Polymer Chain Stiffness \\ on the Behavior of Polymer Nanocomposites \\ José Javier Burgos-Mármol, Óscar Álvarez-Machancoses, and Alessandro Patti \\ J. Phys. Chem. B, Just Accepted Manuscript • Publication Date (Web): 24 May 2017
}

Downloaded from http://pubs.acs.org on May 25, 2017

\title{
Just Accepted
}

"Just Accepted" manuscripts have been peer-reviewed and accepted for publication. They are posted online prior to technical editing, formatting for publication and author proofing. The American Chemical Society provides "Just Accepted" as a free service to the research community to expedite the dissemination of scientific material as soon as possible after acceptance. "Just Accepted" manuscripts appear in full in PDF format accompanied by an HTML abstract. "Just Accepted" manuscripts have been fully peer reviewed, but should not be considered the official version of record. They are accessible to all readers and citable by the Digital Object Identifier (DOI®). "Just Accepted" is an optional service offered to authors. Therefore, the "Just Accepted" Web site may not include all articles that will be published in the journal. After a manuscript is technically edited and formatted, it will be removed from the "Just Accepted" Web site and published as an ASAP article. Note that technical editing may introduce minor changes to the manuscript text and/or graphics which could affect content, and all legal disclaimers and ethical guidelines that apply to the journal pertain. ACS cannot be held responsible for errors or consequences arising from the use of information contained in these "Just Accepted" manuscripts. 


\title{
Modeling the Effect of Polymer Chain Stiffness on the Behavior of Polymer Nanocomposites
}

\author{
J. Javier Burgos-Mármol, Óscar Álvarez-Machancoses, and Alessandro Patti* \\ School of Chemical Engineering and Analytical Science, The University of Manchester, \\ Sackville Street, Manchester M13 9PL, UK. \\ E-mail: alessandro.patti@manchester.ac.uk.
}




\begin{abstract}
Due to their central role in industrial formulations spanning from food packaging to smart coatings, polymer nanocomposites have been the object of a remarkable attention over the last two decades. Incorporating nanoparticles (NPs) to a polymer matrix modifies the conformation and mobility of the polymer chains at the NP-polymer interface and can potentially provide materials with enhanced properties as compared to pristine polymers. To this end, it is crucial to predict and control the ability of NPs to diffuse and achieve a good dispersion in the polymer matrix. Understanding how to control the NPs' dispersion is a challenging task controlled by the delicate balance between enthalpic and entropic contributions, such as NP-polymer interaction, NP size and shape, and polymer chain conformation. By performing Molecular Dynamics (MD) simulations, we investigate the effect of polymer chains' stiffness on the mobility of spherical NPs that establish weak or strong interactions with the polymer. Our results show a sound dependence of the NPs' diffusivity on the long-range order of the polymer melt, which undergoes an isotropic-to-nematic phase transition upon increasing chain stiffness. This phase transition induces a dynamical anisotropy in the nematic phase, with the NPs preferentially diffusing along the nematic director rather than in the directions perpendicular to it. Not only does this tendency determine the NPs' mobility and degree of dispersion in the polymer matrix, but it also influences the resistance to flow of the polymer nanocomposite when a shear is applied. In particular, to assess the role of the chains' conformation on the macroscopic response of our model PNC, we employ reverse non-equilibrium MD to calculate the zero-shear viscosity in both the isotropic and nematic phases, and unveil a plasticizing effect at increasing chain stiffness when the shear is applied along the nematic axis.
\end{abstract}

\title{
I. Introduction
}

Polymer nanocomposites (PNCs) are fascinating materials comprised of nanoparticles (NPs) dispersed in a polymer matrix. The incorporation of particles with size ranging from $10^{-1} \mathrm{~nm}$ to $10^{2}$ 
$\mathrm{nm}$ has been shown to improve the polymer's performance, including its electrical and thermal conductivity, viscoelasticity, and strength. ${ }^{1-4}$ These and other properties are the macroscopic evidence of mechanisms originating at the nanoscale as a result of a combination of enthalpic and entropic contributions, which control the local rearrangement of polymer chains and NPs and the subsequent formation of new (transient) microdomains. Although the genesis of these mechanisms is in the interactions established at the NP-polymer interface, their effect extends to the microscale, and has a dramatic impact on the macroscopic response of the material. ${ }^{5}$ Enthalpic interactions (generally dominant ${ }^{6,7}$ ) are often controlled by modifying the NP's surface, for instance with grafted polymeric ligands, ${ }^{8-10}$ whereas entropic interactions strongly depend on the relative size of polymer chains and NPs as well as on their intrinsic geometry. These tendencies, however, are not of general validity as revealed by the incompatible conclusions of independent experimental studies, especially when assessing the role of interactions on polymer chain and NP mobility. ${ }^{11-14}$

Investigating the ability of NPs to diffuse in a polymer melt is especially relevant to gauge their tendency towards either dispersion or aggregation. Additionally, the NPs' mobility can influence the dynamics of polymer chains so deeply that existing properties, such as the toughness of the material, ${ }^{15}$ are deeply changed. The connection between the dynamics of NPs and polymer chains at the nanoscale and the macroscopic behavior of a PNC is especially evident above the glass transition temperature $\left(T_{g}\right)$, where NPs smaller than the polymer radius of gyration $R_{g}$, due to their enhanced mobility, are particularly effective in dissipating energy and thus toughening the material. ${ }^{15,16}$

The dynamics at such small length scales has a remarkable effect on the macroscopic response of the material, including its rheology. The rheological properties of PNCs have been recently assessed in terms of the interactions established between NPs and polymer chains, ${ }^{21-24}$ the nature of the chain attachment to the NP's surface, ${ }^{25}$ degree of NPs' dispersion, ${ }^{26}$ NP's size ${ }^{27}$ and concentration. ${ }^{28}$ In particular, in a previous study, we showed that NPs smaller than the $R_{g}$ can reduce the viscosity of the polymer melt and hence act as plasticizers, while particles larger than $R_{g}$ act as thickening agents and significantly increase it. ${ }^{27}$ Adding micrometer-sized particles to a 
polymer always increases its viscosity as predicted by the Einstein law, valid in the dilute limit: $\eta=\eta_{p}(1+a \phi)$, where $\eta_{p}$ is the viscosity of the pure polymer, $\phi$ the volume fraction of the particles, and the constant $a=2.5$ the Einstein coefficient or intrinsic viscosity. In the semi-dilute regime, where the volume fraction of the particles is larger, a second order correction is applied: $\eta=\eta_{p}\left(1+a \phi+b \phi^{2}\right)$, where $4.375 \leqslant b \leqslant 6.25 .^{29-32}$ These simple analytical expressions cannot be applied to polymers incorporating nanosized particles, whose viscosity shows a non-trivial dependence on a number of enthalpic and entropic contributions, ${ }^{22}$ and a strong correlation with the specific interface area between polymer chains and NPs. ${ }^{33}$ Nevertheless, molecular modeling allows to switch off many of these contributions by coarse-graining the host polymer and guest NPs as well as their interactions. The coarse-grained (CG) models usually employed to describe the behavior of a polymer drastically reduce the number of its degrees of freedom (e.g. chemical details), while maintaining the main physics underpinning its structural, dynamical, and rheological properties.

In this work, we investigate the effect of chain stiffness on the NPs' and polymer chains' mobility as well as on the shear viscosity of the PNC. By applying simulation and theory, Yethiraj and coworkers analyzed the diffusion of tracers in a melt of frozen and mobile chains at different degrees of chain stiffness. ${ }^{34}$ While the tracer diffusivity results unaffected by the chain rigidity when the chains are frozen, it significantly decreases with increasing chain stiffness in a melt of mobile chains. According to these results, the authors conclude that the tracer diffusion would strongly depend on the local chain dynamics rather than on the chain conformation. We should notice that the tracer's size, being comparable to the monomer's size, is perhaps too small to perceive the effects of a change in chain conformational rigidity and, although it could perfectly model, for instance, molecular diffusion in a polymer, cannot straightaway be employed to reliably reproduce the dynamics of realistic NPs, whose size is usually in the order of $R_{g}$ or larger. The practical relevance of understanding the effect of the chain rigidity is not limited to PNCs, but extends, for instance, to natural macromolecules, such as DNA and proteins, whose diffusion and/or self-diffusion in crowded media controls many relevant biological functions. We observe that increasing the degree 


\section{Model}

To investigate the dynamics of NPs and polymer chains and the viscosity of the PNC, we employ a CG model, where several atoms are grouped together in a simplified manner to reduce the number of interaction sites and then speed up calculations. We stress that coarse-graining a polymer system by disregarding the chemical details of the polymer chains implies relevant approximations that introduce an element of uncertainty at the very small scale, that typical of chemical bond lengths. Nevertheless, CG models have a striking advantage over more sophisticated models: they are able to approach length and time scales currently inaccessible to atomistic models. Because here we investigate phenomena that develop over the nanoscale and do not aim to reproduce the behavior 
of a specific polymer, we employ a CG model that is able to unveil the structure, dynamics and viscosity of a generic homopolymer consisting of linear unentangled chains. ${ }^{33,43-46}$ In the last fifteen years, several CG models have been developed to reproduce the properties of specific polymer systems, including poly(vinyl alcohol), ${ }^{47}$ polyethylene, ${ }^{48-51}$ polystyrene, ${ }^{52}$ and polypropylene. ${ }^{51}$ Although these models are very different from each other in terms of strategy and purpose, they all aim to map the atomistic system of reference into a coarser mesoscopic model that neglects the chemical characteristics of the chains. We refer the interested reader to these works for additional details or to dissipative particle dynamics in case of problems embracing larger length scales and involving hydrodynamics. ${ }^{53-55}$

In particular, our systems contain $N_{n}=80$ spherical NPs in a melt of $N_{c}=4000$ linear polymer chains consisting of $l=10$ beads connected to each other by bond length and bond bending energy terms. Consequently, the total number of beads in the system is $N_{t}=40080$. The diameter and mass of the polymer beads are, respectively, $\sigma$ and $M$, which are the unit length and unit mass of our systems. By contrast, the NP diameter is $\sigma_{n}=3 \sigma$ and its mass $M_{n}=27 M$. A visual representation of the model chains and NPs employed in our simulations is given in Fig. 1.

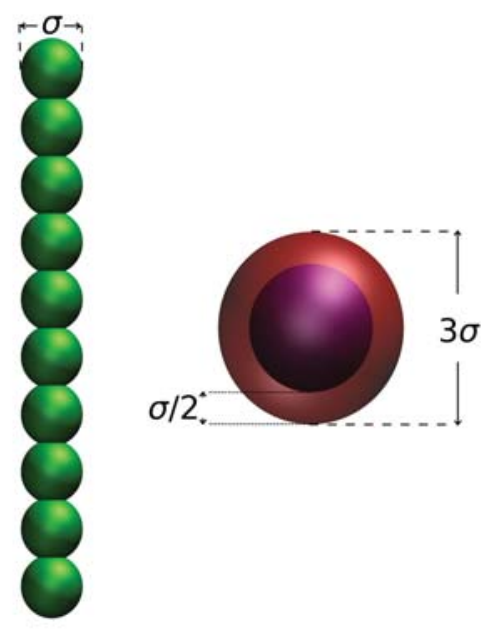

Figure 1: Model polymer chain made of $l=10$ beads with diameter $\sigma$. The red sphere of diameter $\sigma_{n}=3 \sigma$ represents a typical NP dispersed in the polymer matrix, with the hard core highlighted in purple, and the thickness of the soft shell measuring $\sigma / 2$.

All non-bonded interactions are modeled via a modified Lennard-Jones (LJ) potential, $U_{\alpha \beta}$, that 
reads 56

$$
U_{\alpha \beta}(r)= \begin{cases}4 \epsilon_{\alpha \beta}\left[\left(\frac{\sigma}{r-R_{\alpha \beta}}\right)^{12}-\left(\frac{\sigma}{r-R_{\alpha \beta}}\right)^{6}\right]+U_{\alpha \beta}^{0}\left(R_{\alpha \beta}+r_{\alpha \beta}^{c}\right) & \text { if } r<R_{\alpha \beta}+r_{\alpha \beta}^{c} \\ 0 & \text { if } r \geq R_{\alpha \beta}+r_{\alpha \beta}^{c}\end{cases}
$$

Here $\alpha$ and $\beta$ refer to chain beads or NPs and $r$ is the bead-to-bead distance. The energy parameter, $\epsilon_{\alpha \beta}$, depends on the specific pair of beads. In particular, the interaction between two chain beads or monomers $(m)$ is taken as the model unity energy, $\epsilon_{m m}=\epsilon$, and employed to define the reduced temperature, $T^{*} \equiv k_{B} T / \epsilon$, where $k_{B}$ is the Boltzmann constant and $T$ the absolute temperature. The reduced interaction strength between two NPs, $\epsilon_{n n}^{*}=\epsilon_{n n} / \epsilon$, is constant and set to 1 , whereas the reduced NP-monomer interaction strength, $\epsilon_{m n}^{*}=\epsilon_{m n} / \epsilon$, is a simulation parameter assuming the values 1,3 , or 10 . Similarly, the following units are introduced: $t^{*}=t / \tau$, where $t^{*}$ is the reduced time and $\tau=\sigma \sqrt{M / \epsilon}$; and $\eta^{*}=\eta \sigma^{3} \tau^{-1} / \epsilon$, where $\eta^{*}$ is the reduced viscosity. The above modified LJ potential is horizontally and vertically shifted by the amount $R_{\alpha \beta}$ and $U_{\alpha \beta}^{0}\left(R_{\alpha \beta}+r_{\alpha \beta}^{c}\right)$, respectively, where $R_{\alpha \beta}$ and $r_{\alpha \beta}^{c}$ depend on the bead pair, and the vertical shift cancels the potential at the cutoff. In particular, NPs are modeled as core-shell spheres comprising a central hard core surrounded by a soft shell of thickness $\xi_{n} / \sigma=1 / 2$ (Fig. 1). Polymer beads are considered soft spheres without a hard core, namely $R_{m m}=0$. According to these definitions, it follows that $R_{n n}=\sigma_{n}-\sigma$ and $r_{n n}^{c}=2^{1 / 6} \sigma$. Similarly to a Weeks-Chandler-Andersen (WCA) potential, $r_{n n}^{c}$ is defined in such a way that NP-NP interactions are purely repulsive. By contrast, the interaction between monomers and NPs may or may not contain an attractive component, depending on the system studied, with $R_{m n}=\left(\sigma_{n}-\sigma\right) / 2$ in all the cases.

Bonded interactions between adjacent chain beads are described by the sum of two contributions: the LJ potential provided in Eq. (1) that prevents chain overlaps, and the Finitely Extensible Nonlinear Elastic (FENE) potential that prevents chain breaking. The FENE potential reads:

$$
V_{F E N E}(r)=-\frac{1}{2} k R_{0}^{2} \ln \left[1-\left(\frac{r}{R_{0}}\right)^{2}\right]
$$

where $k \sigma^{2} / \epsilon=30$ and $R_{0} / \sigma=1.5$ are the intensity and range of the interaction, respectively. 
The chain stiffness is incorporated via a harmonic potential describing the fluctuations of the bond angle:

$$
U_{\text {bending }}=K_{\theta}\left(\theta-\theta_{0}\right)^{2}
$$

where $\theta$ and $\theta_{0}=\pi$ are, respectively, the bond angle and its equilibrium value, whereas $K_{\theta}$ is the interaction strength and measures the degree of chain stiffness. In particular, $K_{\theta}^{*}=K_{\theta} / \epsilon$ is the reduced chain stiffness and a simulation parameter varying between 0 and 100 . In summary, the complete force field employed to describe the bonded interactions is

$$
U_{\text {bonding }}(r, \theta)=V_{F E N E}(r)+U_{\text {bending }}(\theta)+U_{m m}(r) \text {, }
$$

with $R_{m m}=0$ and $r_{m m}^{c} / \sigma=2^{1 / 6}$.

\section{Simulation Methodology}

We performed MD simulations to equilibrate the systems and analyze their structure and dynamics, and reverse non-equilibrium MD (RNEMD) simulations to determine their shear viscosity. ${ }^{57,58}$ All the simulations were carried out using the Large-scale Atomic/Molecular Massively Parallel Simulator (LAMMPS) $)^{42}$ with a timestep $\delta t=10^{-3} \tau$. Each system has been equilibrated at the same temperature $T^{*}=1$ in cubic boxes with periodic boundaries and side length $L=40.776 \sigma$. The initial configurations consisted of chains and NPs sequentially arranged as in a lattice network that were allowed to relax gradually to the desired temperature by employing a Nosé-Hoover thermostat. This produced a completely random distribution resembling an isotropic polymer melt incorporating NPs and provided the starting configuration for the production runs. Equilibrium MD simulations have been performed in the $N V T$ (canonical) ensemble, at constant $N_{t}, T$ and simulation box volume, $V$. The interaction between chain beads and NPs has been adjusted by tuning the simulation parameters $\epsilon_{m n}$ and $r_{m n}^{c}$. In particular, four sets of parameters define these interactions, and read $\left(\epsilon_{m n}^{*}, r_{m n}^{c *}\right)=\left(1,2^{1 / 6}\right),(1,2.5),(3,2.5)$, and $(10,2.5)$. The first set of parameters 
refers to an athermal system, where monomer-monomer, monomer-NP and NP-NP interactions are identical, whereas the three remaining sets produce attractive monomer-NP interactions and will be identified by the value of $\epsilon_{m n}^{*}$. In addition, the degree of chain stiffness is adjusted by modifying the parameter $K_{\theta}$, which assumes the values $K_{\theta}^{*}=0,1,2,5,10,20,50$, and 100 .

To assess the main structural features of the systems studied, we calculated $(i)$ the mean-square radius of gyration, $\left\langle R_{g}^{2}\right\rangle$, of the polymer chains, (ii) their mean-square end-to-end distance, $\left\langle R_{e e}^{2}\right\rangle$, (iii) the pair correlation functions, $g_{\alpha \beta}(r)$, and (iv) the nematic order parameter, $S_{2}$. More specifically, the mean-square radius of gyration and end-to-end distance read

$$
\left\langle\boldsymbol{R}_{g}^{2}\right\rangle=\frac{1}{l^{2}} \sum_{i=1}^{l}\left\langle\left(\boldsymbol{r}_{i}-\boldsymbol{r}_{c m}\right)^{2}\right\rangle
$$

and

$$
\left\langle\boldsymbol{R}_{e e}^{2}\right\rangle=\sum_{i=1}^{l-1} \sum_{j=1}^{l-1}\left\langle\boldsymbol{b}_{i} \cdot \boldsymbol{b}_{j}\right\rangle,
$$

where $\langle\ldots\rangle$ indicate ensemble average, $\boldsymbol{r}_{i}$ and $\boldsymbol{r}_{c m}$ are the position vectors of the monomer $i$ in a given chain and the center of mass of this chain, respectively, and $\boldsymbol{b}_{i}=\boldsymbol{r}_{i+1}-\boldsymbol{r}_{i}$ is the bond vector between the chain beads $i$ and $i+1$. The radial distribution function for a generic pair of beads $\alpha$ and $\beta$ is computed as

$$
g_{\alpha \beta}(r)=\frac{1}{\rho_{\beta}} \frac{\left\langle n_{\beta}(r)\right\rangle_{\alpha}}{V_{\text {shell }}(r)},
$$

where $\rho_{\beta}=n_{\beta} / V$ is the number density of beads $\beta$ in the simulation box; $\left\langle n_{\beta}(r)\right\rangle_{\alpha}$ is the average number of beads $\beta$ at a distance $r$ from a bead $\alpha$; and $V_{\text {shell }}(r)$ is the volume of a shell centered on $\alpha$ and inner and outer radii $r \pm \Delta r / 2$, with $\Delta r$ the shell thickness.

In order to unambiguously distinguish an isotropic from a nematic phase, we estimate the nematic order parameter, $S_{2}$, which quantifies the alignment of the principal molecular axis of the polymer chains with the nematic director of the bulk phase: 


$$
S_{2}=\frac{1}{2}\left\langle 3\left|\hat{\boldsymbol{u}}_{i} \cdot \hat{\boldsymbol{n}}\right|^{2}-1\right\rangle
$$

where $\hat{\boldsymbol{u}}_{i}=\left(\boldsymbol{r}_{i, l}-\boldsymbol{r}_{i, 1}\right) /\left|\boldsymbol{r}_{i, l}-\boldsymbol{r}_{i, 1}\right|$ is the unit orientation vector of chain $i$. The definition of $\hat{\boldsymbol{u}}_{i}$ is arbitrary and such that its ensemble average is zero in an isotropic collection of chains: $\left\langle\hat{\boldsymbol{u}}_{i}\right\rangle=0$. In particular, $S_{2}$ and $\hat{\boldsymbol{n}}$ are, respectively, the largest eigenvalue and the corresponding unit eigenvector of the following second-rank tensor

$$
\boldsymbol{Q}=\frac{1}{N_{c h}} \sum_{i=1}^{N_{c}}\left(\frac{3}{2} \hat{\boldsymbol{u}}_{i} \otimes \hat{\boldsymbol{u}}_{i}-\frac{1}{2} \boldsymbol{I}\right)
$$

with $\otimes$ the dyadic product and $\boldsymbol{I}$ the unit second-rank tensor. All the above properties have been averaged over $2 \cdot 10^{3}$ configurations and every $5 \cdot 10^{3}$ timesteps.

To assess the mobility of polymer chains and NPs, we estimate the diffusion coefficients from the slope of the mean squared displacement (MSD) at long times. As far as the polymer is concerned, both the MSD of the chains' center of mass and that of the chain beads are calculated in order to assess their relative mobility and separately compare it to that of NPs. In the isotropic phase, the MSD is calculated as

$$
\left\langle\Delta r_{\alpha}^{2}\right\rangle=\left\langle\left[\boldsymbol{r}_{\alpha}(t)-\boldsymbol{r}_{\alpha}\left(t_{0}\right)\right]^{2}\right\rangle
$$

with $t_{0}$ the initial time of the trajectory of bead $\alpha$. The resulting diffusion coefficient reads

$$
D=\frac{1}{6} \lim _{t \rightarrow \infty} \frac{\partial\left\langle\Delta r_{\alpha}^{2}\right\rangle}{\partial t}
$$

By contrast, in the nematic phase, we separately estimate the MSD along $\hat{\boldsymbol{n}}$ and in the plane perpendicular to $\hat{\boldsymbol{n}}$ by evaluating the projections $r_{\alpha, \|}=\boldsymbol{r}_{\alpha} \cdot \hat{\boldsymbol{n}}$ and $r_{\alpha, \perp}=\sqrt{r_{\alpha}^{2}-r_{\alpha, \|}^{2}}{ }^{59}$ Consequently, the parallel, $D_{\|}$, and perpendicular, $D_{\perp}$, diffusion coefficients read:

$$
D_{\|}=\frac{1}{2} \lim _{t \rightarrow \infty} \frac{\partial\left\langle\Delta r_{\alpha, \|}^{2}\right\rangle}{\partial t},
$$


and

$$
D_{\perp}=\frac{1}{4} \lim _{t \rightarrow \infty} \frac{\partial\left\langle\Delta r_{\alpha, \perp}^{2}\right\rangle}{\partial t}
$$

The shear viscosity of our model PNCs is estimated by employing the RNEMD method originally proposed by Müller-Plathe for LJ fluids ${ }^{57,58}$ and later extended to polymers. ${ }^{60}$ Here we review the main features of this method and refer the interested reader to these works for details. The shear viscosity, $\eta$, links the transverse momentum flux, say, in the $z$ direction, $J_{z}\left(p_{x}\right)$, to the velocity gradient $\partial v_{x} / \partial z$, according to the following equation:

$$
J_{z}\left(p_{x}\right)=-\eta \frac{\partial v_{x}}{\partial z}
$$

where $p_{x}$ is the momentum in the $x$ direction, with $\hat{\boldsymbol{e}}_{x} \perp \hat{\boldsymbol{e}}_{z}$. In RNEMD, the flux is generated by an unphysical momentum transfer across the system. More specifically, a given equilibrated system is first replicated in one direction, say $z$, to obtain an elongated orthorhombic simulation box and then relaxed to level out the density fluctuations. The box is then divided in 20 slabs along the largest dimension $z$. The chain bead with the largest negative momentum in the $x$ direction is selected from the top slab, and the chain bead with the largest positive momentum in the $x$ direction is selected from the central slab. The velocities of the two beads in the $x$ direction are swapped and, as a consequence, momentum is transferred across the slabs. Because the selected beads have the same mass, this velocity swap will not alter the total momentum and kinetic energy, both of which are conserved. It should be noticed that, due to their relative low concentration in the system as compared to the chain beads, pairs of NP beads are never selected, although the momentum transfer will still affect their velocities. The resulting momentum flux can be calculated as

$$
J_{z}\left(p_{x}\right)=\frac{p_{x}}{2 t A}
$$

where $t$ is the length of the simulation, $A$ the area of each slab, and the factor 2 arises because the momentum transfer occurs from one slab to the other along two possible directions. This flux 
generates a velocity gradient $\partial v_{x} / \partial z$ that can be considered linear if the flux, controlled by the period, $W \delta t$, of the velocity swap, is not too large. The flow velocity in each slab is determined by averaging over the velocities of all the beads in that slab and the final slope $-\left\langle\partial v_{x} / \partial z\right\rangle$ equals the shear rate $\dot{\gamma}$. By increasing the velocity swap period, the shear rate decreases. ${ }^{60}$ The shear viscosity is then calculated as

$$
\eta=\frac{p_{x}}{2 t A \dot{\gamma}}
$$

As far as the isotropic systems are concerned, the shear viscosity can be evaluated by averaging out six contributions to the momentum flux:

$$
\begin{array}{r}
\eta_{\text {iso }}=-\frac{1}{6}\left(\frac{J_{x}\left(p_{y}\right)}{\left\langle\partial v_{y} / \partial x\right\rangle}+\frac{J_{y}\left(p_{x}\right)}{\left\langle\partial v_{x} / \partial y\right\rangle}+\frac{J_{x}\left(p_{z}\right)}{\left\langle\partial v_{z} / \partial x\right\rangle}+\right. \\
\left.\frac{J_{z}\left(p_{x}\right)}{\left\langle\partial v_{x} / \partial z\right\rangle}+\frac{J_{y}\left(p_{z}\right)}{\left\langle\partial v_{z} / \partial y\right\rangle}+\frac{J_{z}\left(p_{y}\right)}{\left\langle\partial v_{y} / \partial z\right\rangle}\right) .
\end{array}
$$

By contrast, nematic systems are aligned in the direction of $\hat{\boldsymbol{n}}$ and therefore the value of $\eta$ is not unique, but depends on the orientation of the nematic director. In particular, we can define three independent viscosity coefficients when the nematic director is: 1) parallel to the shear flow, 2) parallel to the velocity gradient, and 3) perpendicular to both. ${ }^{61}$ As a consequence, assuming that $\hat{\boldsymbol{n}} \| \hat{\boldsymbol{e}}_{z}$, these terms are determined by:

$$
\begin{aligned}
& \eta_{1}=-\frac{1}{2}\left(\frac{J_{x}\left(p_{z}\right)}{\left\langle\partial v_{z} / \partial x\right\rangle}+\frac{J_{y}\left(p_{z}\right)}{\left\langle\partial v_{z} / \partial y\right\rangle}\right), \\
& \eta_{2}=-\frac{1}{2}\left(\frac{J_{z}\left(p_{x}\right)}{\left\langle\partial v_{x} / \partial z\right\rangle}+\frac{J_{z}\left(p_{y}\right)}{\left\langle\partial v_{y} / \partial z\right\rangle}\right), \\
& \eta_{3}=-\frac{1}{2}\left(\frac{J_{x}\left(p_{y}\right)}{\left\langle\partial v_{y} / \partial x\right\rangle}+\frac{J_{y}\left(p_{x}\right)}{\left\langle\partial v_{x} / \partial y\right\rangle}\right) .
\end{aligned}
$$




\section{Results}

Due to its effect on the translational entropy of the polymer chains, the degree of chain stiffness is expected to have a relevant impact on the distribution of the NPs in the polymer melt and on their mobility, being both aspects determinant for the macroscopic response of a PNC. In this section, we investigate how changing chain flexibility can induce an isotropic-to-nematic $(I / N)$ phase transition, which provokes an intriguing anisotropic NP's diffusion and influences the rheological behavior of the systems studied. The $I / N$ phase transition, visually portrayed in Fig. 2 and determined by mapping the nematic order parameter $S_{2}$, is characterized by a chain reorientation along a common nematic director, $\hat{\boldsymbol{n}}$.
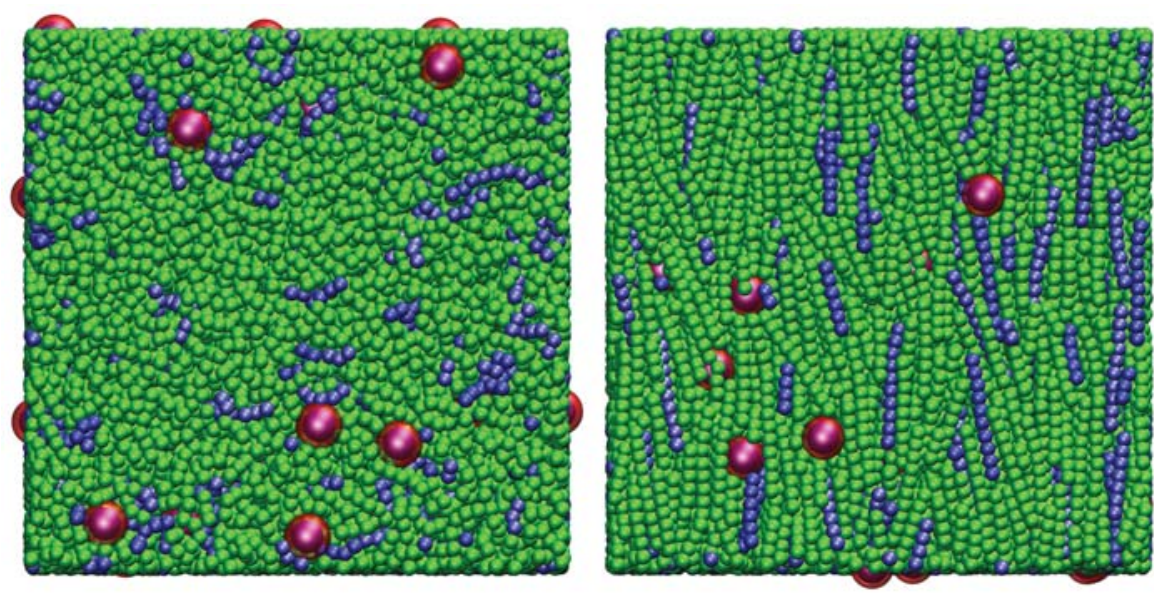

Figure 2: Equilibrium configurations of polymer melts incorporating spherical NPs at degree of chain stiffness $K_{\theta}^{*}=1$ (left) and $K_{\theta}^{*}=100$ (right), with $\epsilon_{m n}^{*}=1$. Polymer beads are in green, with some chains highlighted in blue to better appreciate their conformation, and NPs are in red, with their hard cores highlighted in purple.

The degree of dispersion of NPs within the polymer matrix has been analyzed by estimating the monomer-monomer, chains' center of mass-center of mass, monomer-NP, and NP-NP radial distribution functions (RDFs), or equivalently, $g_{m m}, g_{c c}, g_{m n}$, and $g_{n n}$, respectively. Both $\epsilon_{m n}$ and $K_{\theta}$ do not display any remarkable effect on the distribution of the polymer chain beads, with the $g_{m m}$ functions (not shown here) exhibiting the typical behavior of a dense fluid with relatively strong correlations at short distances and fast decay to 1. Similarly, the distribution of the centers of mass of the chains (Fig. 3) is not significantly affected by $\epsilon_{m n}$, but can locate the isotropic to nematic 
phase transition, which can be appreciated from the appearance of a clear peak at $r / \sigma \simeq 1.4$ and $K_{\theta}^{*}>10$. The functions $g_{c c}$ at $K_{\theta}^{*}=0$ (blue dotted lines in Fig. 3) show that fully flexible chains are folded, implying that their center of mass is mostly surrounded by beads of the same chain. Therefore, we conclude that the most probable distance between two centers of mass is $r \simeq 2 R_{g}$. At increasing degrees of stiffness, but still in the isotropic phase, the polymer chains start to arrange into unfolded configurations with their center of mass lying outside the chain contour length and potentially overlapping with the center of mass of neighboring chains. This picture is supported by the larger values displayed by $g_{c c}(r / \sigma<1)$ at $K_{\theta}^{*}=1$ (solid black lines in Fig. 3) as compared to those obtained at $K_{\theta}^{*}=0$ (blue dotted lines in the same figure). At $K_{\theta}^{*} \geqslant 10$, with the chains completely unfolded and their monomers roughly aligned to each other along a common direction, the probability to observe the chains' center of mass at a mutual distance $r / \sigma<1$ decreases again and becomes negligible at $K_{\theta}^{*}=100$ (red dashed lines in Fig. 3). The latter condition implies that the chains' center of mass are aligned with the same chain beads, and the monomer-monomer interactions prevent them from approaching each other at a distance smaller than $r / \sigma=1$. The monomer-NP RDFs (Fig. 4) show a non-negligible dependence on the interaction strength as the increasing height of the first peak with $\epsilon_{m n}$ indicates (note the different scale in the vertical axis). In this case, the stronger attraction between NPs and chain beads results into an enhanced local chain adsorption on the NP's surface, especially evident at $K_{\theta}^{*}=1$ as flexible chains better adapt to the the NP's curvature.

As far as the NP-NP RDFs are concerned (Fig. 5), both $K_{\theta}$ and $\epsilon_{m n}$ have an important effect on the degree of dispersion of NPs. Specifically, under athermal conditions (Fig. 5a) the polymer chains are less likely to be found in the interparticle space, thus promoting a partial NP's clustering that is favored at larger degrees of chain stiffness (red dashed line in Fig. 5a). The clusters observed in these systems are generally elongated and approximately oriented along the nematic director (inset in Fig. 5a). At slightly attractive interactions $\left(\epsilon_{m n}^{*}=1\right)$, this effect is much weaker than under athermal conditions, with the main peak of $g_{n n}(r)$ reducing significantly and a second peak forming at $r / \sigma \simeq 4.1$. While the former peak disappears at increasing NP-monomer interactions, 

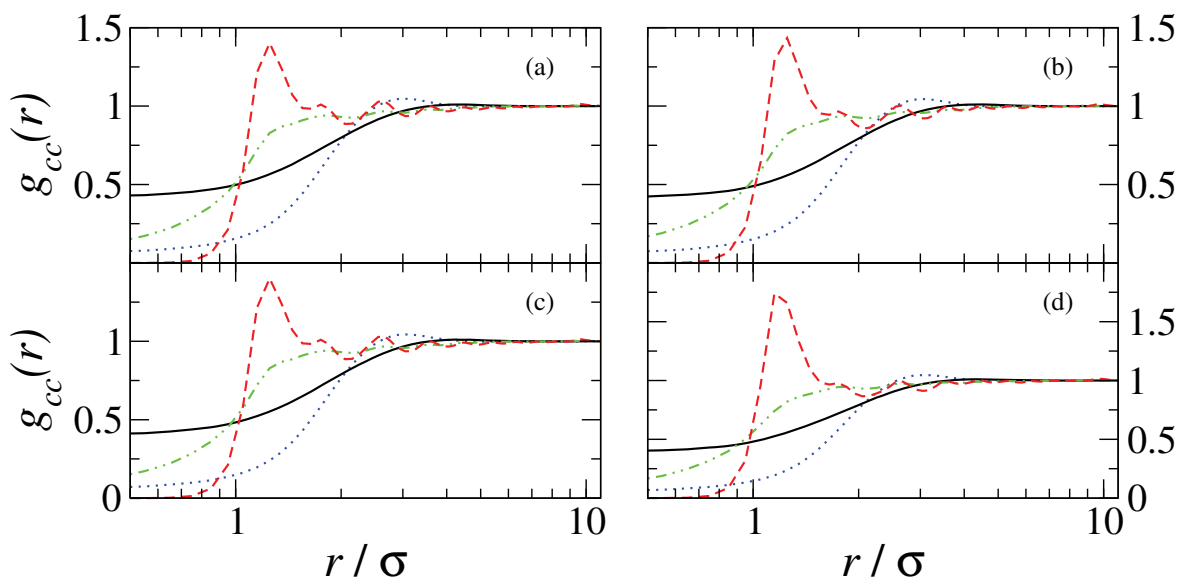

Figure 3: Chains' center of mass-center of mass radial distribution functions under athermal conditions (a) and at $\epsilon_{m n}^{*}=1$ (b), 3 (c), and 10 (d). In each frame, dotted blue, solid black, dash-dotted green, and dashed red curves refer to degrees of chain stiffness $K_{\theta}^{*}=0,1,10$ and 100, respectively. Note the different scale in the vertical axis of frame (d).
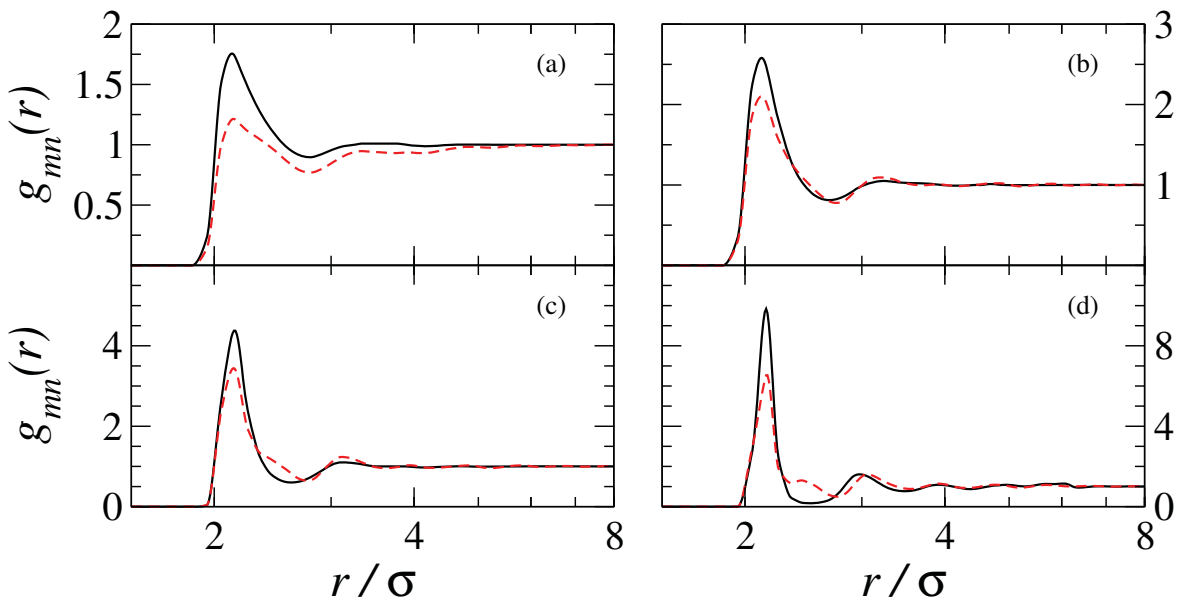

Figure 4: Monomer-NP radial distribution functions under athermal conditions (a) and at $\epsilon_{m n}^{*}=1$ (b), 3 (c), and 10 (d). In each frame, solid black and dashed red curves refer to degrees of chain stiffness $K_{\theta}^{*}=1$ and 100 , respectively.

the latter becomes more and more relevant, establishing a transition from string-like clusters of NPs to a homogeneous dispersion of NPs. In particular, larger monomer-NP attractions promote the presence of polymer chains on the NP surface, thus favoring the homogeneous dispersion of NPs, as observed at $\epsilon_{m n}^{*}=1$ and $\epsilon_{m n}^{*}=3$, in Figs. 5b and 5c, respectively. By further increasing the attraction between chains and NPs, the formation of binary clusters, consisting of polymer chains intercalating between close neighboring NPs, is promoted (Fig. 5d). Because flexible chains can better adapt to the NP's curvature, this tendency is more clearly detected at small degrees of chain 

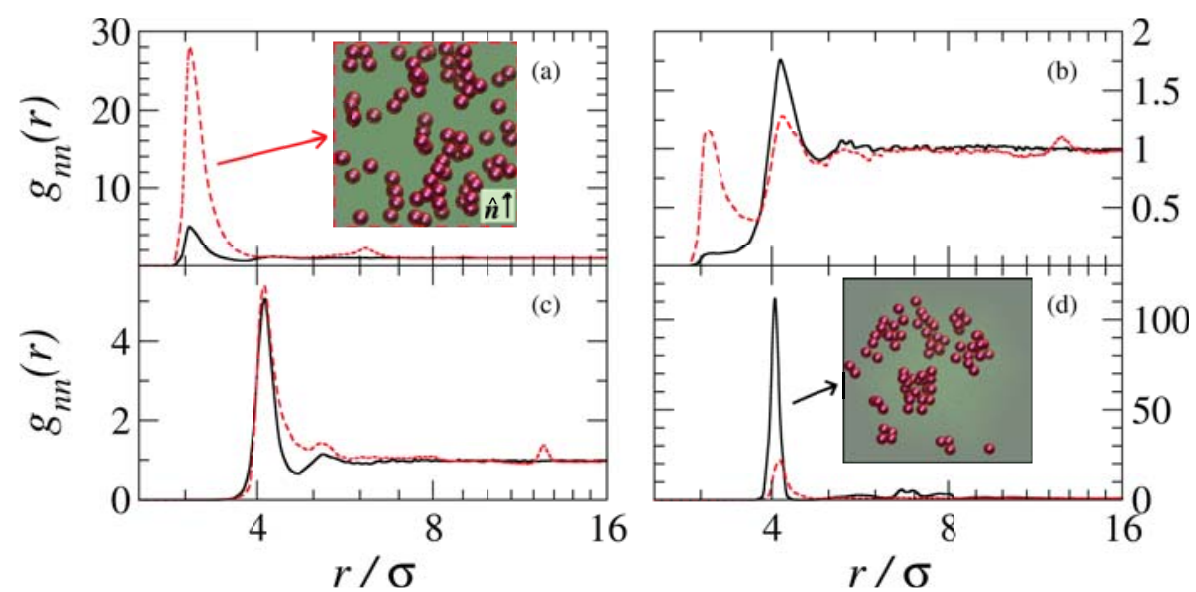

Figure 5: Radial distribution functions of NP-NP beads under athermal conditions (a) and at $\epsilon_{m n}^{*}=$ 1 (b), 3 (c), and 10 (d). In each frame, solid black and dashed red curves refer to degrees of chain stiffness $K_{\theta}^{*}=1$ and 100, respectively. Insets in frames (a) and (d) provide typical configurations of model PNCs at $K_{\theta}^{*}=1$ and 100, respectively.

stiffness, where the binary clusters are roughly spherical (see inset in Fig. 5d). Therefore, by tuning the strength of the NP-monomer interactions and degree of chain stiffness, we are able to observe a spectrum of local structures spanning from string-like clusters of NPs to chain-NP binary aggregates. We stress that only at moderate values of $\epsilon_{m n}$ (for instance, at $\epsilon_{m n}^{*}=3$ ) a homogeneous distribution of NPs, not particularly influenced by the degree of chain stiffness, can be achieved.

The local morphology of the polymer at the bead-scale can be assessed by estimating the polymer chain's characteristic lengths, for instance the radius of gyration, $R_{g}$, and end-to-end distance, $R_{e e}$. Both parameters are reported in Fig. 6 as a function of $K_{\theta}$ and $\epsilon_{m n}$. While the strength of monomer-NP interaction has practically no effect on $R_{g}$ and $R_{e e}$, the constant regulating the chain stiffness deeply affects the conformation of the polymer chains especially up to $K_{\theta}^{*}=10$, above which $R_{g}$ and $R_{e e}$ saturate to the steady value of $2.8 \sigma$ and $8.7 \sigma$, respectively. Moreover, the standard deviations of $R_{g}$ and $R_{e e}$, indicated by the vertical bars in Fig. 6, show that the distribution of the polymer chains' conformation is very narrow at large $K_{\theta}$, but increasingly wider at small $K_{\theta}$, and it is not affected by $\epsilon_{m n}$.

It is convenient to calculate the persistence length of the polymer chains at different degrees of chain stiffness. The persistence length, $p$, is defined as the length over which the correlations along the tangent to the chain bonds are lost. From the ideal chain model, the persistence length is 


$$
p=\frac{1}{\ln \langle\cos \theta\rangle},
$$

where $\theta$ is the bond angle defined in Section II to measure the bending potential. It should be noticed that this definition assumes that the correlation decay of the chain angles is an exponential function of their distance along the chain. This assumption is strictly true only for ideal chains as such orientational autocorrelation function has been reported to follow a power law in self avoiding chain models. ${ }^{62,63}$ Therefore, the results reported in Fig. 7 should be considered on a mere qualitative basis to address how the rigidity of the chain is influenced by the isotropic-tonematic transition. Nevertheless, the above mentioned definition of the persistence length can be still applied to characterize the local chain rigidity. ${ }^{64}$ The dependence of the persistence length on the chain stiffness is shown in Fig. 7. Although we estimated $p$ at the chain ends and central 
segments, its values were practically independent of the position in the chain, most likely because our chains are relatively short, as well as of the monomer-NP interaction. When plotting $p=p\left(K_{\theta}^{*}\right)$ in Fig. 7, we identify two distinct sets of values that can be fitted by a power law of the type $p=A K_{\theta}^{\lambda}$, with reduced parameters $A^{*}=1.98$ and $\lambda=1.02$ at $K_{\theta}^{*} \leqslant 5$ (isotropic phase) and $A^{*}=2.38$ and $\lambda=0.97$ at $K_{\theta}^{*} \geqslant 20$ (nematic phase). The point $p\left(K_{\theta}^{*}=10\right)$ lies in between the two power laws.

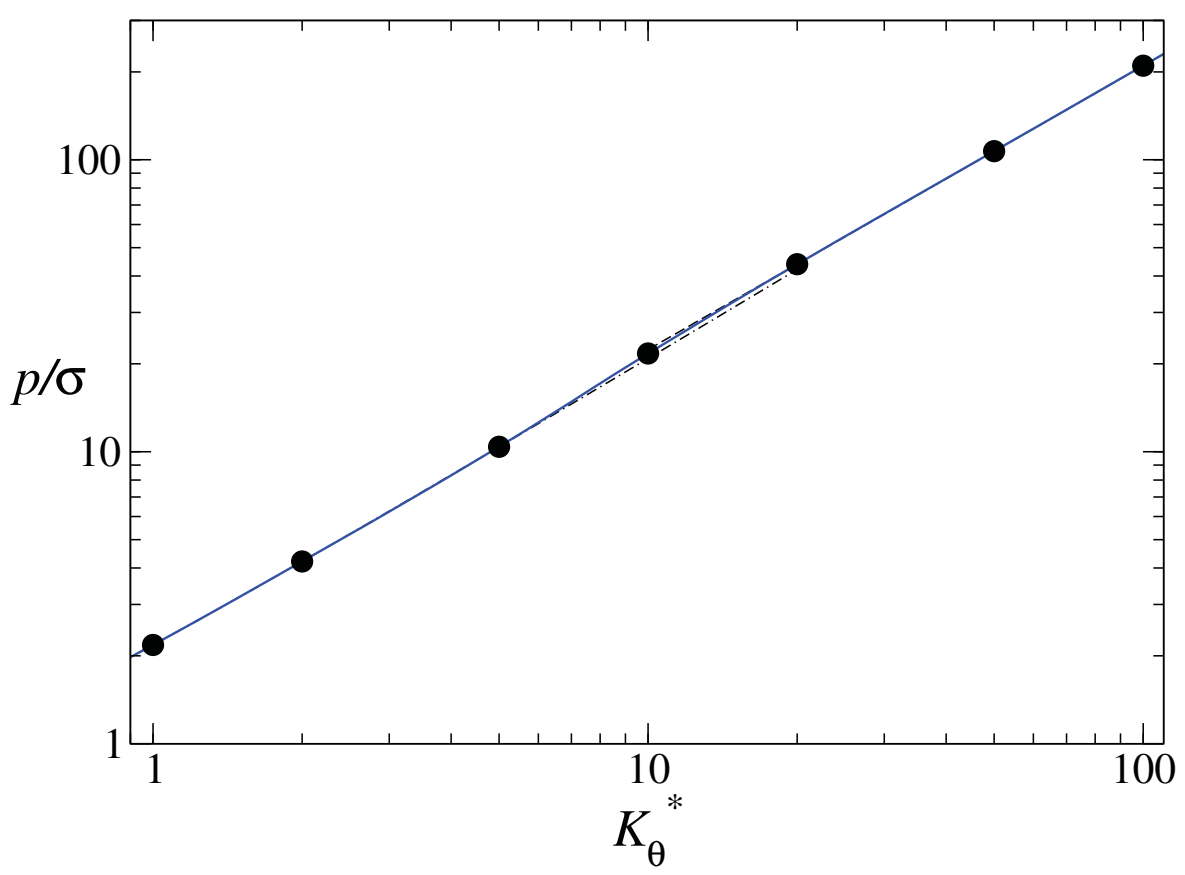

Figure 7: Persistence length of the polymer chains at different degrees of chain stiffness. The dashed and dashed-dotted lines are power law fittings to the isotropic and nematic phases, respectively. The solid line is a guide for the eyes. Error bars are smaller than the symbol size.

Such a substantial change in the flexibility of the polymer chains drives the transition from the isotropic to the nematic phase, which can be located by calculating the nematic order parameter, $S_{2}$, given in Fig. 8 as a function of $K_{\theta}$. The nematic order parameter varies in the range [0,1], with $S_{2}=0$ in a fully disordered (isotropic) phase, and $S_{2}=1$ in a perfectly aligned nematic phase. Flexible chains are weakly ordered, their segments do not show any relevant orientation, and, consequently, the corresponding order parameter remains very low, as generally observed in an isotropic fluid. By contrast, at $K_{\theta}^{*}>5$, the polymer chains start to orient along a common nematic director and $S_{2}$ increases up to approximately 0.9 , a value that is consistent with the 
theoretical predictions for systems of semiflexible polymer chains. ${ }^{65,66}$ More generally, the $\mathrm{I} / \mathrm{N}$ phase transition has been reported to be intimately related to the ratio $l / p .{ }^{64,67}$ Consequently, the value of $K_{\theta}$ at which we observe the $\mathrm{I} / \mathrm{N}$ phase transition, would not be conserved after modifying the length or the density of chains in the system. We also notice that stiff rod-like chains might not form a nematic phase if their length-to-diameter ratio is not large enough, regardless of the value of $K_{\theta}$, and an isotropic-to-smectic phase transition would be rather observed. ${ }^{38}$

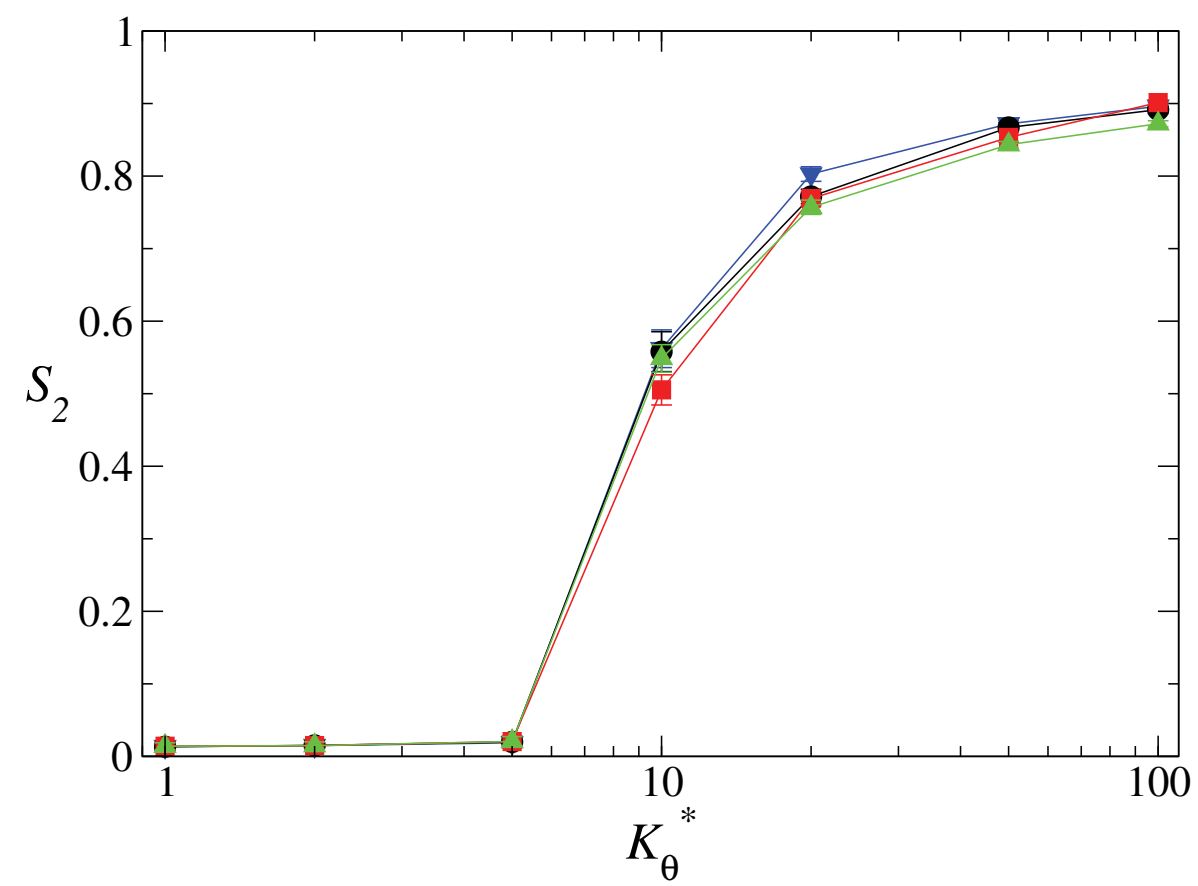

Figure 8: Nematic order parameter as a function of the degree of polymer chain stiffness at different monomer-NP interaction strengths: athermal $(\boldsymbol{\nabla}), \epsilon_{m n}^{*}=1(\bullet), 3(\square)$, and $10(\Delta)$. Solid lines are a guide for the eye and vertical bars represent standard deviations.

Not only does the increase of chain stiffness induce the isotropic-to-nematic phase transition, but it also significantly influences the mobility of both polymer chains and NPs. To appreciate these effects, it is convenient to decouple the MSD of chains and NPs along the parallel and perpendicular directions to the nematic director $\hat{\boldsymbol{n}}$, here indicated as $\left\langle\Delta r_{\|}^{2}\right\rangle$ and $\left\langle\Delta r_{\perp}^{2}\right\rangle$, respectively. In Figs. 9 and 10 , we show the MSDs of chain monomers and chains' center of mass, respectively, at $K_{\theta}^{*}=10$ and 100 , and $\epsilon_{m n}^{*}=3$. At the these values of chain stiffness, the system is in the nematic phase. For comparison, we also show the MSDs at $K_{\theta}^{*}=1$, where the system is isotropic as well as the dynamics therein. As a general tendency, the long-time diffusive dynamics of chains and 


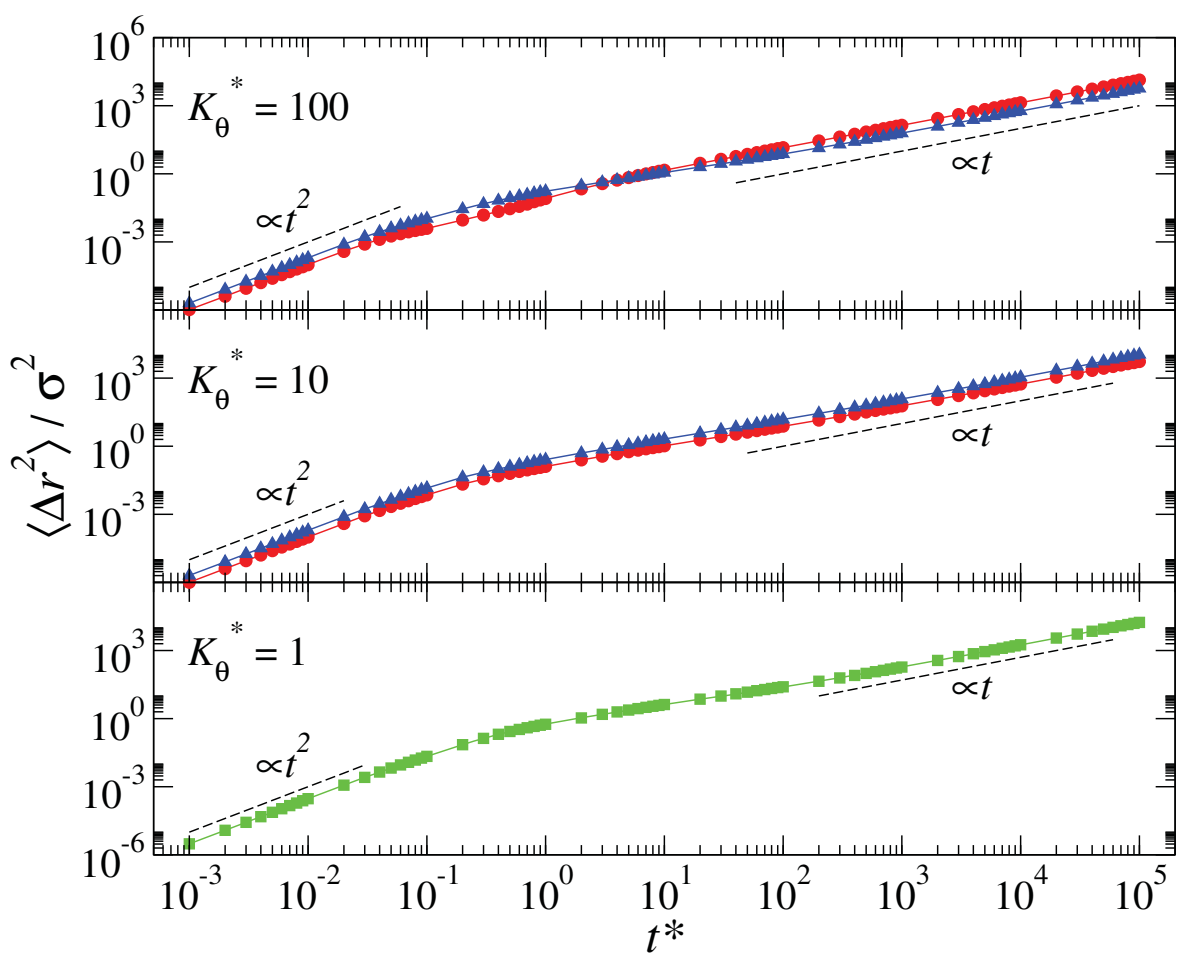

Figure 9: MSD of chain monomers at $\epsilon_{m n}^{*}=3$ and degrees of chain stiffness $K_{\theta}^{*}=1$ (bottom frame), 10 (middle frame), and 100 (top frame). Squares refer to the total MSD calculated in the isotropic phase, whereas circles and triangles to the MSD parallel and perpendicular to the nematic director, respectively. Dashed lines indicate the dependence of the MSD on time at short and long timescales.

beads is very similar, especially for particularly stiff chains, whose segmental motion is strongly correlated to the mobility of its center of mass. Both in the isotropic and nematic phases, the beads move generally faster than the whole chain in the ballistic regime, where $\left\langle\Delta r^{2}\right\rangle \propto t^{2}$, but this difference becomes almost negligible at long times. The alignment of the chains at $K_{\theta}^{*}>$ 5 determines substantial differences in the dynamics along $\hat{\boldsymbol{n}}$ and perpendicularly to it. More specifically, in systems with high degrees of stiffness, $\left\langle\Delta r_{\|}^{2}\right\rangle$ shows a very smooth crossover from the ballistic to the diffusive regime, whereas $\left\langle\Delta r_{\perp}^{2}\right\rangle$ displays a subdiffusive dynamics at intermediate times as a result of the cage effect hampering the transverse displacement of monomers and chains. The slowing down of the transverse dynamics, being more evident at increasing $K_{\theta}^{*}$, produces a crossover between $\left\langle\Delta r_{\|}^{2}\right\rangle$ and $\left\langle\Delta r_{\perp}^{2}\right\rangle$ at $t^{*} \simeq 4$ for the chain monomers (Fig. 9) and $t^{*} \simeq 1$ for the polymer chains (Fig. 10). This crossover, which is associated to the anisotropic density distribution 


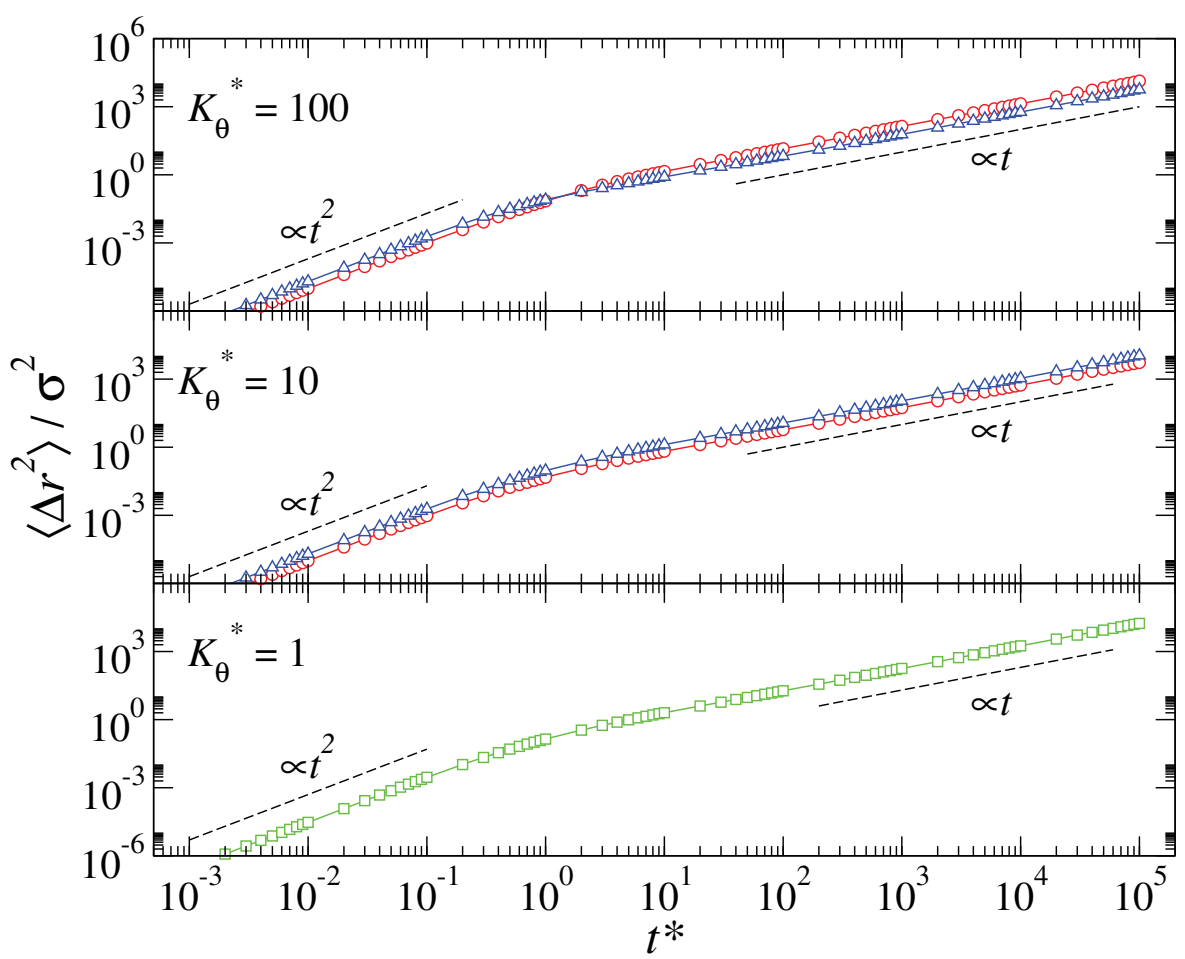

Figure 10: MSD of polymer chains at $\epsilon_{m n}^{*}=3$ and degrees of chain stiffness $K_{\theta}^{*}=1$ (bottom frame), 10 (middle frame), and 100 (top frame). Squares refer to the total MSD calculated in the isotropic phase, whereas circles and triangles to the MSD parallel and perpendicular to the nematic director, respectively. Dashed lines indicate the dependence of the MSD on time at short and long timescales.

of the polymer chains, was also observed by computer simulations in colloidal suspensions of stiff rod-like and spherical particles ${ }^{68}$ and experimentally in suspensions of semi-flexible rod-like viruses. ${ }^{69}$ However, at $K_{\theta}^{*}=10$, despite the nematic order parameter being larger than at smaller degrees of stiffness, there is no crossover between the two components of the MSD. This suggests that the polymer matrix forms a weak nematic phase, where the polymer chains are not strongly oriented and are not stiff enough to hamper the NPs' mobility in the plane perpendicular to the nematic director. In other words, motion of NPs in planes perpendicular to $\hat{\boldsymbol{n}}$ is not significantly affected by moderate degrees of chain stiffness. The dynamics of monomers and chains at $\epsilon_{m n}^{*}=1$ and 10 (MSDs not shown here) are, respectively, slightly faster and slower than those shown in Figs. 9 and 10, but follow similar tendencies.

The dynamics of NPs is strongly affected by the interaction with the polymer chains as can be 


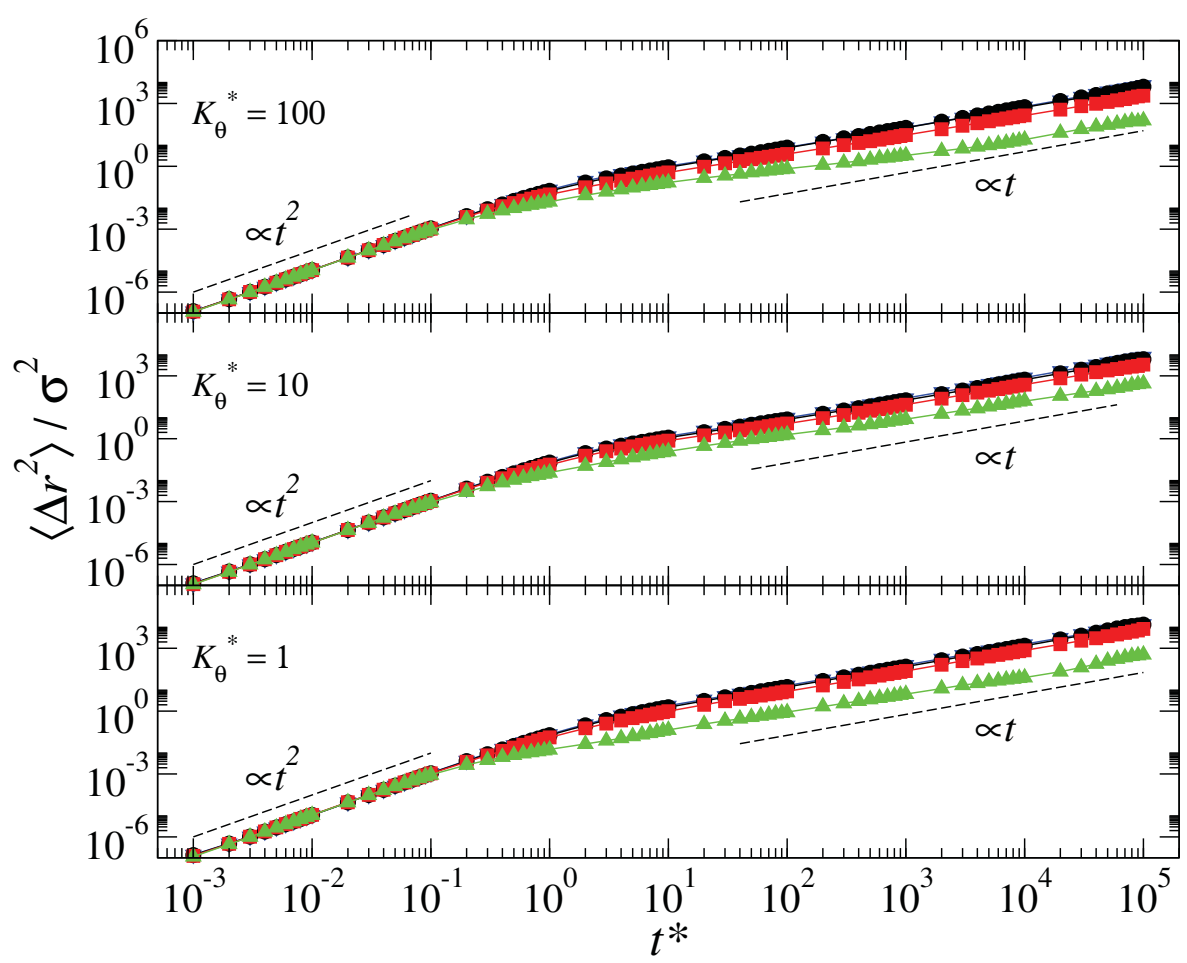

Figure 11: MSD of spherical NPs in a polymer melt at three different degrees of chain stiffness: $K_{\theta}^{*}=1$ (bottom frame), 10 (middle frame), and 100 (top frame). The NP-monomer interaction strength is athermal $(\nabla), \epsilon_{m n}^{*}=1(\bullet), 3(\square)$, and $10(\Delta)$. Dashed lines indicate the dependence of the MSD on time at short and long timescales.

inferred from Fig. 11, where the NPs' isotropic MSDs are shown as a function of $K_{\theta}$ and $\epsilon_{m n}$. In particular, at a given chain stiffness, the stronger the monomer-NP interaction is, the slower the NP's diffusion in the melt. This results in an additional time decade needed to displace a NP by its diameter when the monomer-NP interaction increases from $\epsilon_{m n}^{*}=1$ (black circles in Fig. 11) to $\epsilon_{m n}^{*}=10$ (green triangles in the same figure). At $t^{*}>1$, the MSDs display a linear dependence on time as the NPs enter the diffusive regime for all the monomer-NP interactions studied except $\epsilon_{m n}^{*}=10$, at which the diffusive regime is achieved only at much larger time scales $\left(t^{*} \gtrsim 10^{4}\right)$ while a subdiffusive behavior is displayed at intermediate timescales $\left(1<t^{*} \lesssim 10^{4}\right)$. The latter interaction determines a subdiffusive regime with the NPs slowing down and $\left\langle\Delta r^{2}\right\rangle \propto t^{\beta}$, with $\beta$ ranging from 0.7 at $K_{\theta}^{*}=100$ to 0.8 at $K_{\theta}^{*}=1$. This behavior is in good agreement with the structure observed in Fig. 5d, that shows a sticking effect between NPs intermediated by polymer chains. 
At long time scales, we calculated the diffusion coefficients to better clarify the combined effect of $K_{\theta}$ and $\epsilon_{m n}$ on the NP's mobility in the polymer matrix. The results are shown in Fig. 12 for different monomer-NP interactions and degrees of chain stiffness.

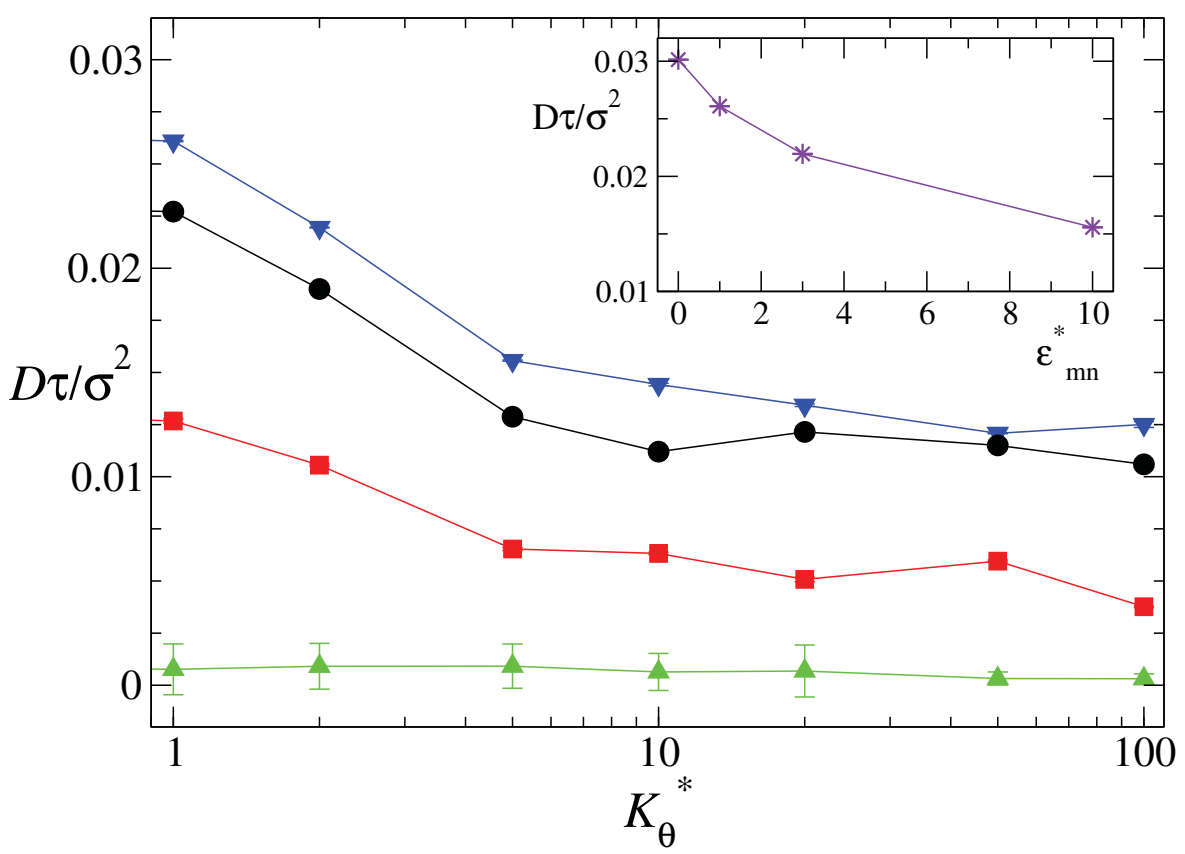

Figure 12: Diffusion coefficient of NPs as a function of the polymer chain stiffness at different NP-monomer interaction strengths: athermal $(\nabla), \epsilon_{m n}^{*}=1(\bullet), 3(\square)$, and $10(\Delta)$. The inset shows the dependence of the diffusion coefficient on the NP-monomer interaction strength at $K_{\theta}^{*}=0$.

As expected from the tendency detected in the above MSDs, the NP diffusion coefficients decrease at increasing monomer-NP interaction. In particular, the average ratio between the diffusion coefficients at $\epsilon_{m n}^{*}=1$ and those at $\epsilon_{m n}^{*}=10$ is in the order of $10^{2}$. In the isotropic phase, that is at $K_{\theta}^{*} \leqslant 5$, the NP's diffusion is faster than that in the nematic phase, especially when the interaction with the polymer chains is relatively weak. Increasing chain stiffness reduces the isotropic diffusivity by a factor of 2 at athermal interactions and $\epsilon_{m n}^{*}=1$ and $\epsilon_{m n}^{*}=3$. At stronger interactions, the diffusion is equally hampered over the whole range of $K_{\theta}^{*}$ studied, with $D \tau / \sigma^{2} \simeq 10^{-4}$. In the nematic phase, the global (isotropic) diffusion appears to be practically unaffected by the degree of chain stiffness. However, a more careful analysis, where $D_{\|}$and $D_{\perp}$, rather than the isotropic diffusivity, are analyzed, reveals an intriguing scenario, with the diffusion in the direction of $\hat{\boldsymbol{n}}$ significantly faster than that in the plane perpendicular to it. This tendency is observed in Fig. 13, 
which gives the diffusion coefficients in the directions parallel and perpendicular to $\hat{\boldsymbol{n}}$ as a function of the chain stiffness at different NP-monomer interactions. Furthermore, it can be observed that at increasing chain stiffness, with the polymer chains acquiring a significant long-range orientational order, $D_{\|}$also increases by up to a factor of 3 as compared to $D_{\perp}$. Similar ratios were observed by Park and Sung in polymer melts incorporating smaller NPs. ${ }^{70}$ Strong monomer-NP interactions drastically hamper the NP's mobility in the transverse direction to $\hat{\boldsymbol{n}}$, but do not prevent the parallel diffusion, which is favored by the chains' alignment. The two contributions to the NPs' mobility is such that the isotropic diffusion coefficient, $D=\left(D_{\|}+2 D_{\perp}\right) / 3$, remains basically constant over the whole range of degrees of chain stiffness studied here. We also note that the NPs diffuse with no preferential direction when the chain monomers are attracted to NPs, but show an improved mobility along the nematic axis in the athermal system. The MSDs $\left\langle\Delta r_{\|}^{2}\right\rangle$ and $\left\langle\Delta r_{\perp}^{2}\right\rangle$ showed the same crossover at $K_{\theta}^{*}=10$ and at larger values of $K_{\theta}$ under athermal conditions (not shown here). By contrast, in systems with $\epsilon_{m n}^{*}=1$ (not shown here) and $\epsilon_{m n}^{*}=3$ (Fig. 12), the MSDs show this crossover only at $K_{\theta}^{*} \geqslant 20$. Therefore, the diffusive behavior of the NPs is strongly dependent on the monomer-NP interactions at $K_{\theta}^{*}=10$, which corresponds to a weak nematic phase. As far as the systems with $\epsilon_{m n}^{*}=10$ are concerned, their dynamics were too slow for the parallel and perpendicular components of the MSD to reach a full diffusive regime in the timescales covered by our simulations.

The analysis of the MSDs and associated diffusivities highlights the crucial role played by enthalpic and entropic contributions in determining the NPs' distribution in a polymer melt. In particular, properly tuning the chemistry of the NP's surface, for instance by its functionalization with ligand molecules, and the flexibility of the polymer chains, for instance by incorporating double bounds or side groups, allows one to predict and control the (anisotropic) dynamics of NPs in the polymer matrix and hence minimize their often undesired tendency towards clustering. Understanding the mechanisms that explain the structural and dynamical properties of a polymer-based material enormously contributes to shed light on the fundamental laws that govern its microscopic behavior. Nevertheless, it is likewise crucial to provide a more general picture, able to interpret 


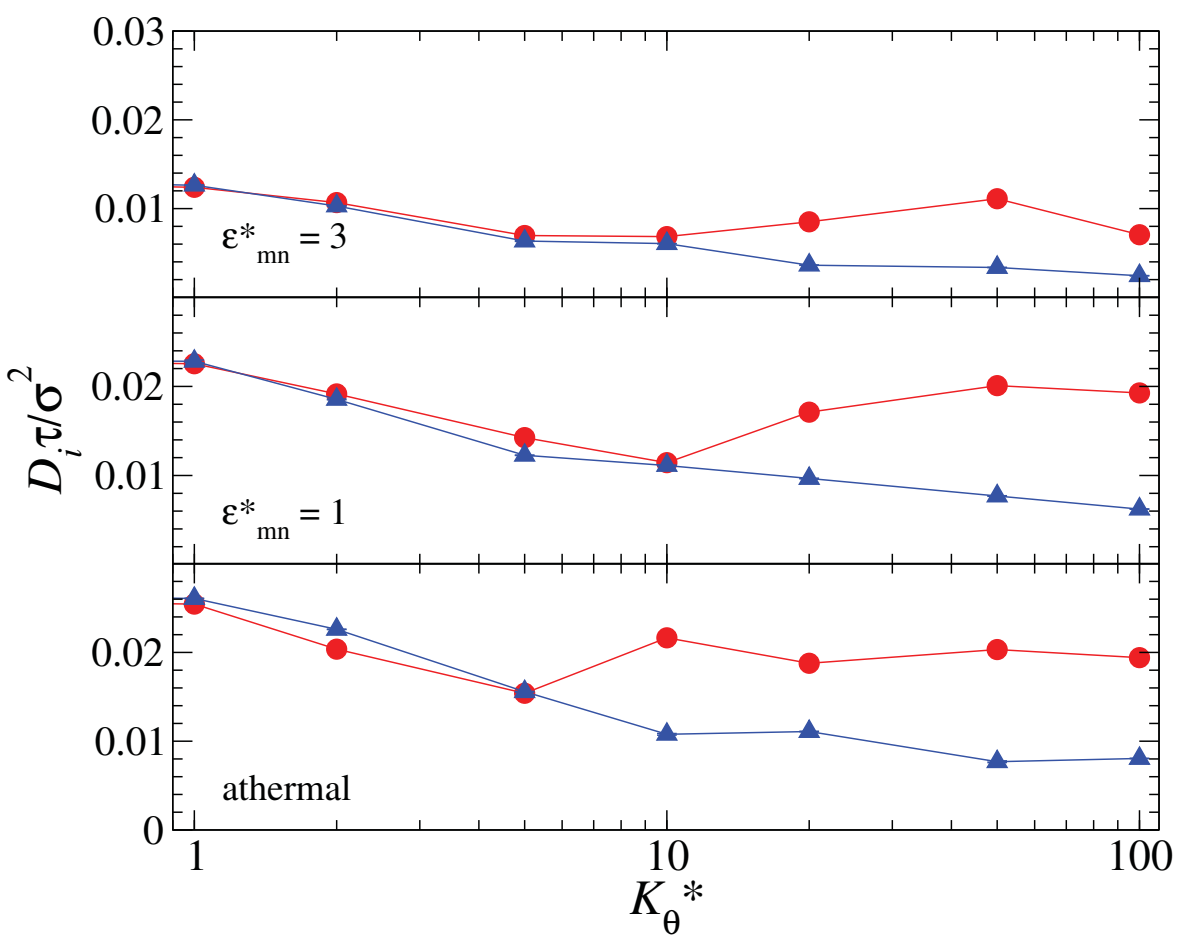

Figure 13: Parallel $\left(D_{\|}, \bigcirc\right)$ and perpendicular $\left(D_{\perp}, \boldsymbol{\Delta}\right)$ diffusion coefficients of NPs with respect to the nematic director as a function of the degree of chain stiffness. Lines are a guide for the eye.

the impact of such laws on the response of the material at the macroscopic scale. To this end, we now investigate the shear rheology of our model PNC by employing the RNEMD method. More specifically, we study the shear viscosity as a function of the shear rate $\dot{\gamma}$ and then extrapolate the shear viscosity at infinitesimally low shear rates to address its dependence on the strength of monomer-NP interactions and degree of chain stiffness.

In Fig. 14, we show the shear viscosity as a function of the shear rate $\dot{\gamma}$ at different degrees of chain stiffness. In the isotropic phase (top frame), two separate viscoelastic regimes can be observed: a linear (Newtonian) regime at shear rates $\dot{\gamma} \tau<0.01$ and a non-linear regime at larger shear rates, where the system exhibits shear-thinning. The solid line is a fitting to the model by Daivis et al. for molecular fluids, which reads $\eta=\eta_{0}-A \dot{\gamma}^{2},{ }^{71}$ with $\eta_{0}$ and $A$ the zero-shear viscosity and a fitting parameter, respectively. In particular, the reduced values obtained for this particular system are $\eta_{0}^{*}=2.37$ and $A^{*}=501$. By contrast, nematic systems show different responses depending on the relative orientation of the nematic director, the shear flow and the velocity gradient. More specifically, three different responses are shown in the bottom frame of 


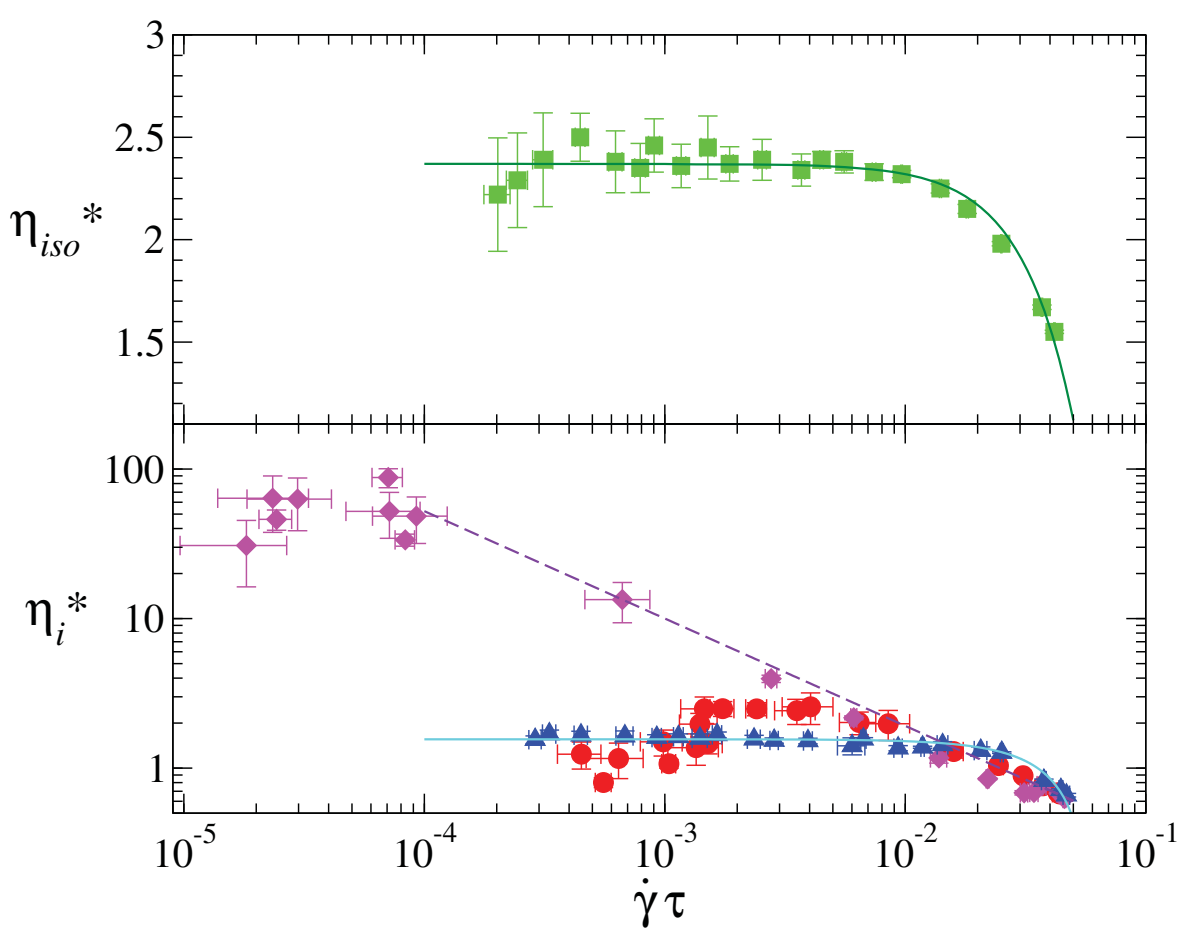

Figure 14: Viscosity as a function of the shear rate for an isotropic (top frame, $\epsilon_{m n}^{*}=1, K_{\theta}^{*}=1$ ) and a nematic phase (bottom frame, $\epsilon_{m n}^{*}=1, K_{\theta}^{*}=100$ ). Squares $(\square)$, circles $(\bullet)$, diamonds $(\diamond)$, and triangles $(\boldsymbol{\Delta})$ refer to $\eta_{i s o}, \eta_{1}, \eta_{2}$, and $\eta_{3}$, respectively. The solid lines are a fit to the function $\eta=\eta_{0}-A \dot{\gamma}^{2}$, and the dashed line is a fit in the non-linear regime of $\eta_{2}$ to the function $B \dot{\gamma}^{-\alpha}$ (see text).

Fig. 14, where, at small shear rates, $\eta_{2}$ is one or two orders of magnitude larger than $\eta_{1}$ and $\eta_{3}$. The solid line is a fit of $\eta_{3}$ to Daivis model, with $\eta_{0}^{*}=1.55$ and $A^{*}=443$. By contrast, $\eta_{1}$ and $\eta_{2}$ do not fit well Daivis model, but can be very well adjusted to a power law in the non-linear regime of the type $\eta=B \dot{\gamma}^{-\alpha}$, with $B^{*}=0.07$ and $\alpha=0.72$ (dashed line). These parameters fit both $\eta_{1}$ and $\eta_{2}$ at shear rates $\dot{\gamma} \tau>10^{-2}$ and $\dot{\gamma} \tau>10^{-4}$, respectively. Moreover, the three curves tend to converge at larger values of $\dot{\gamma}$, which indicates that the melt is probably reoriented in the direction of the shear. A power law that is able to simultaneously fit both $\eta_{1}$ and $\eta_{2}$ is not surprising as the fitting parameter $\alpha$ is expected to increase with the polymer chain length, ${ }^{60,72,73}$ which is constant in this study. While the value of $\alpha$ obtained in these simulation studies is roughly about 0.7 , reptation theory, assuming the polymer confined in a tube-like region and following a reptation dynamics, predicts a rather larger value of $\alpha=1.5{ }^{74}$ The difference is most probably due to the Rouse dynamics followed by the short chains employed in our simulations as compared to the entangled 
chains described by the reptation theory.

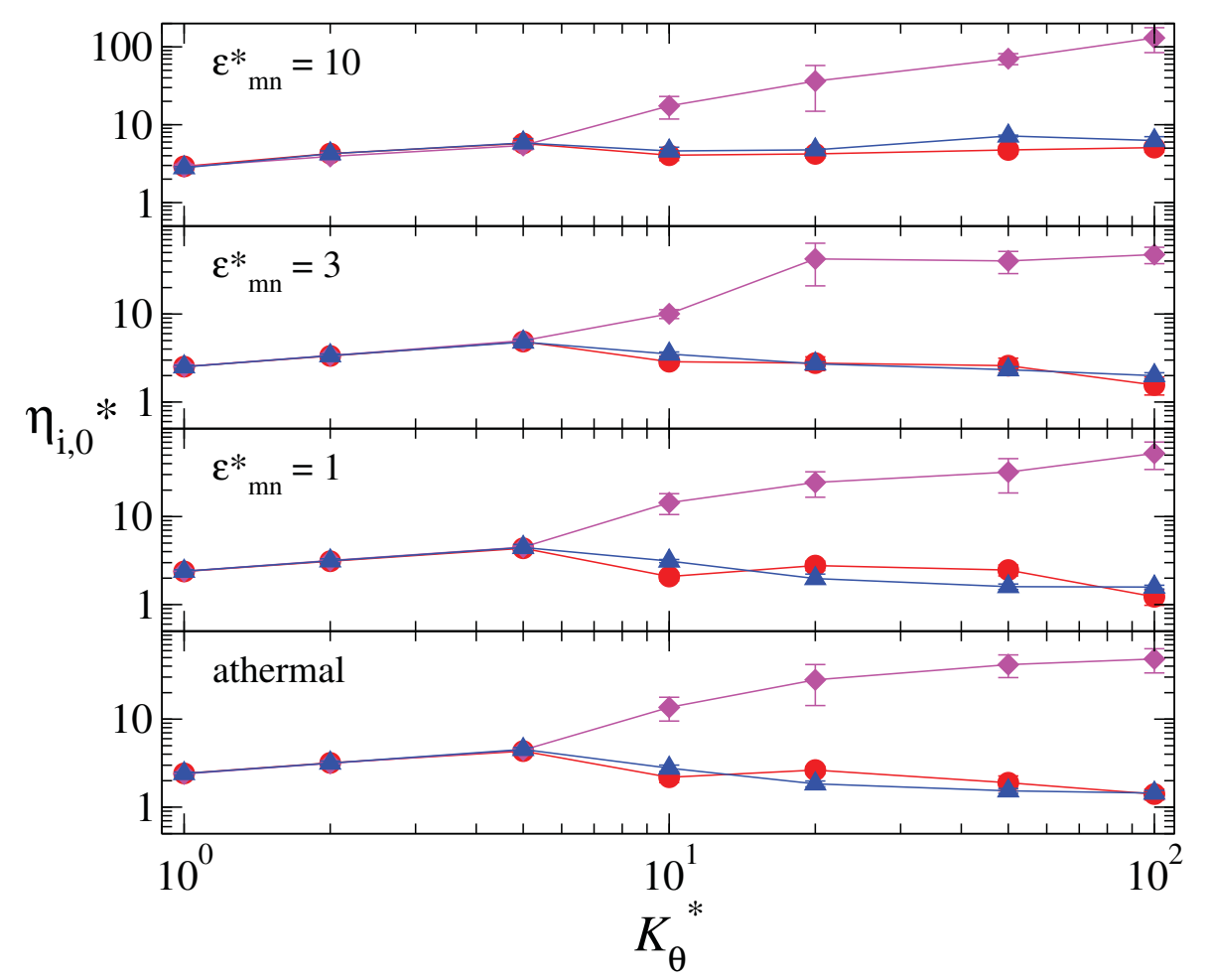

Figure 15: Zero-shear viscosity as a function of the degree of polymer chain stiffness for different monomer-NP interactions. Circles $(\bullet)$, diamonds $(\diamond)$, and triangles $(\boldsymbol{\Delta})$ refer to $\eta_{1}, \eta_{2}$, and $\eta_{3}$, respectively. Solid lines are guides for the eye.

In the linear viscoelastic regime, where $\dot{\gamma} \tau<<1$, the zero-shear viscosity $\eta_{0}$ is extrapolated at $\dot{\gamma} \rightarrow 0$ and plotted as a function of the degree of chain stiffness in Fig. 15. In the isotropic phase $\left(K_{\theta}^{*} \leqslant 5\right), \eta_{0}$ displays a monotonic growth at the four monomer-NP interactions studied. This can be explained by the increase in $R_{g}$ and the number of entanglements per chain with the degree of stiffness. ${ }^{33,75}$ At larger degrees of stiffness, the melt forms a nematic phase and the viscosity is described by three different curves, ${ }^{61}$ depending on the direction of the shear. While $\eta_{1}(\bigcirc)$ and $\eta_{3}(\Delta)$ show a decreasing tendency with respect to the viscosity values in the isotropic phase, $\eta_{2}$ $(\diamond)$ increases dramatically by up to almost two orders of magnitude. This behavior is qualitatively the same in all the systems studied, regardless of the monomer-NP interactions. However, only systems with $\epsilon_{m n}^{*}=10$ display significantly larger values of $\eta_{1}, \eta_{2}$, and $\eta_{3}$ than the others. This quantitatively small influence of low monomer-NP interactions on the viscosity can be justified 
by the low monomer-NP specific interface area, which is roughly proportional to the fraction of polymer chain beads affected by monomer-NP interactions. The larger this amount of contacts is, the more sensitive the viscosity becomes to the the increase in monomer-NP interactions. ${ }^{33}$

\section{Conclusions}

In summary, we have performed extensive MD simulations of polymer melts incorporating spherical NPs and investigated their structure, dynamics, and viscoelastic behavior as a function of polymer chain stiffness at different monomer-NP interaction strength. Increasing the stiffness of the polymer chains promotes a phase transition from a weakly ordered (isotropic) phase to a nematic liquid crystal phase, where the orientationally ordered chains dramatically influence the dynamics and resistance to flow of the whole system. The distribution of NPs is generally homogeneous in the polymer unless the interactions between the former and the latter are relatively weak and the chains particularly stiff. In this case, the stiff chains tend to act as depletants by clustering the NPs together in order to enhance the packing of the system. By contrast, completely flexible chains can better adapt to the NPs' curvature and, as the analysis of the radial distribution functions suggested, favor their dispersion in the polymer matrix. For similar reasons, flexible polymers act as a gluing agent and favor the concentration of particles in small areas in systems with strong monomer-NP interactions, while more rigid chains, which cannot adapt their shape to the NPs' surface, favor the homogeneous dispersion of NPs. The effect of chain stiffness on the dynamics of NPs and polymer chains is dramatically influenced by the isotropic-to-nematic phase transition, promoting the longitudinal over the transverse diffusion of both components. This is especially evident in systems incorporating very stiff chains that establish strong monomer-NP interactions. Under these conditions, the diffusivity of NPs along the nematic director was observed to increase by up to a factor of 3 as compared to their diffusivity in the direction perpendicular to it. Not only does the chains' stiffness determine the mobility of NPs and chains, but also the viscoelastic response of the whole system and its resistance to flow. By performing RNEMD simulations, we estimated the dependence of the shear viscosity on the shear rate and observed a linear viscoelastic regime fol- 
lowed by shear thinning well described by a quadratic decay in the isotropic phase, whereas three different behaviors were obtained in the nematic phase, related to the direction of shear. In the linear regime, the zero-shear viscosity was determined and studied as a function of the degree of chain stiffness. While in the isotropic phase the zero-shear viscosity follows a monotonic growth, the full alignment of the chains in the nematic phase determines a slight reduction of the zero-shear viscosity if the shear flow is parallel to the nematic director. Similarly, if the nematic director is oriented perpendicularly to both the shear flow and the velocity gradient, the zero-shear viscosity also decreases. By contrast, a relevant increase in $\eta_{0}$ is observed when the nematic director is parallel to the velocity gradient.

\section{Acknowledgments}

JJBM acknowledges a postgraduate grant from the University of Manchester (AA02879). The authors would like to acknowledge the assistance given by IT Services and the use of the Computational Shared Facility at The University of Manchester.

\section{References}

(1) Tyan, H.-L.; Liu, Y.-C.; Wei, K.-H. Thermally and Mechanically Enhanced Clay/Polyimide Nanocomposite via Reactive Organoclay, Chem. Mater. 1999, 11, 1942-1947.

(2) Ramanathan, T.; Liu, H.; Brinson, L. C. Functionalized SWNT/Polymer Nanocomposites for Dramatic Property Improvement, J. Polym. Sci., Part B: Polym. Phys. 2005, 43, 2269-2279.

(3) Sanchez-Jiménez, P. E.; Pérez-Maqueda, L. A.; Perejón, A.; Criado, J. M., Nanoclay Nucleation Effect in the Thermal Stabilization of a Polymer Nanocomposite: A Kinetic Mechanism Change, J. Phys. Chem. C 2012, 116, 11797-11807.

(4) Ramanathan, T.; Abdala, A. A.; Stankovich, S.; Dikin, D. A.; Herrera-Alonso, M.; Piner, R. 
D.; Adamson, D. H.; Schniepp, H. C.; Chen, X.; Ruoff, R. S. et al. Functionalized Graphene Sheets for Polymer Nanocomposites, Nat. Nanotech., 2008, 3, 327-331.

(5) Crosby, A. J.; Lee, J.-Y. Polymer Nanocomposites: The "Nano" Effect on Mechanical Properties, Polymer Reviews, 2007, 47, 217-229.

(6) Mackay, M. E.; Tuteja, A.; Duxbury, P. M.; Hawker, C. J.; Horn, B. V.; Guan, Z.; Chen, G.; Krishnan, R. S. General Strategies for Nanoparticle Dispersion, Science 2006, 311, 1740.

(7) Hooper J. B.; Schweizer, K. S. Theory of Phase Separation in Polymer Nanocomposites, Macromolecules 2006, 39, 5133-5142.

(8) Chiu, J. J.; Bumjoon, J. K.; Kramer, E. J.; Pine, D. J. Control of Nanoparticle Location in Block Copolymers, J. Am. Chem. Soc. 2005, 127, 5036-5037.

(9) Zhao, Y.; Thorkelsson, K.; Mastroianni, A. J.; Schilling, T.; Luther, J. M.; Rancatore, B. J.; Matsunaga, K.; Jinnai, H.; Wu, Y.; Poulsen, D.; Frchet, J. M. J.; Alivisatos, A. P.; Xu, T. Small-molecule-directed Nanoparticle Assembly towards Stimuli-responsive Nanocomposites, Nat. Mater. 2009, 8, 979-985.

(10) Ghanbari, A.; Rahimi, M.; Dehghany, J., Influence of Surface Grafted Polymers on the Polymer Dynamics in a Silica-Polystyrene Nanocomposite: A Coarse-Grained Molecular Dynamics Investigation Influence of Surface Grafted Polymers on the Polymer Dynamics in a Silica-Polystyrene Nanocomposite. J. Phys. Chem. C, 2013, 117, 25069-25076.

(11) Rittigstein, P.; Priestley, R. D.; Broadbelt, L. J.; Torkelson, J. M., Model Polymer Nanocomposites Provide an Understanding of Confinement Effects in Real Nanocomposites, Nat. Mater., 2007, 6, 278-282.

(12) Bogoslovov, R. B.; Roland, C. M.; Ellis, A. R.; Randall, A. M.; Robertson, C. G. Effect of Silica Nanoparticles on the Local Segmental Dynamics in Poly(vinyl acetate), Macromolecules 2008, 41, 1289-1296. 
(13) Papon, A. et al., Low-Field NMR Investigations of Nanocomposites: Polymer Dynamics and Network Effects, Macromolecules, 2011, 44, 913.

(14) Schneider, G. J.; Nusser, K.; Neueder, S.; Brodeck, M.; Willner, L.; Farago, B.; Holderer, O.; Briels, W. J.; Richter, D., Anomalous Chain Diffusion in Unentangled Model Polymer Nanocomposites, Soft Matter, 2013, 9, 4336-4348.

(15) Gersappe, D., Molecular Mechanisms of Failure in Polymer Nanocomposites, Phys. Rev. Lett., 2002, 89, 058301.

(16) Shah, D.; Maiti, P.; Jiang, D. D.; Batt, C. A.; Giannelis, E. P., Effect of Nanoparticle Mobility on Toughness of Polymer Nanocomposites, Adv. Mater., 2005, 17, 525-528.

(17) Starr, F. W.; Schrøder, T. B.; Glotzer, S. C., Effects of a Nanoscopic Filler on the Structure and Dynamics of a Simulated Polymer Melt and the Relationship to Ultrathin Film, Phys. Rev. E, 2001, 64, 021802.

(18) Torres, J.A. ; Nealey, P.F. ; de Pablo, J.J., Molecular Simulation of Ultrathin Polymeric Films near the Glass Transition, Phys. Rev. Lett., 2000, 85, 3221.

(19) Bansal, A.; Yang, H.; Li, C.; Cho, K.; Benicewicz, B. C.; Kumar, S. K.; Schadler, L. S., Quantitative Equivalence between Polymer Nanocomposites and Thin Polymer Films, Nature Mater., 2005, 4, 693-698.

(20) Holt, A. P.; Bocharova, V.; Cheng, S.; Kisliuk, A. M.; White, B. T.; Saito, T.; Uhrig, D.; Mahalik, J. P.; Kumar, R.; Imel, A. E. et al. Controlling Interfacial Dynamics: Covalent Bonding versus Physical Adsorption in Polymer Nanocomposites, ACS Nano, 2016, 10, 6843-6852.

(21) Smith G.D., Bedrov D., Li L., Byutner O.; A Molecular Dynamics Simulation Study of the Viscoelastic Properties of Polymer Nanocomposites; J. Chem. Phys., 2002, 117, 94787-9490.

(22) Kalathi, J. T.; Grest, G. S.; Kumar, S. K., Universal Viscosity Behavior of Polymer Nanocomposites, Phys. Rev. Lett., 2012, 109, 198301. 
(23) Chen, Y.; Li, Z.; Wen, S.; Yang, Q.; Zhang, L.; Zhong, C.; Liu, L., Molecular Simulation Study of Role of PolymerParticle Interactions in the Strain-dependent Viscoelasticity of Elastomers (Payne Effect), J. Chem. Phys., 2014, 141, 104901.

(24) Mogurampelly, S.; Sethuraman, V.; Pryamitsyn, V.; Ganesan, V., Influence of NanoparticleIon and Nanoparticle-Polymer Interactions on Ion Transport and Viscoelastic Properties of Polymer Electrolytes, J. Chem. Phys., 2016, 144, 154905.

(25) Hattemer, G.D.; Arya, G., Viscoelastic Properties of Polymer-Grafted Nanoparticle Composites from Molecular Dynamics Simulations, Macromolecules, 2015, 48, 1240-1255.

(26) Tang, L.-C.; Wan, Y.-J.; Yan, D.; Pei, Y.-B; Zhao, L.; Li, Y.-B.; Wu, L.-B.; Jiang, J.-X.; Lai, G.-Q., The Effect of Graphene Dispersion on the Mechanical Properties of Graphene/Epoxy Composites, Carbon, 2013, 60, 16-27.

(27) Patti, A.; Molecular Dynamics of Spherical Nanoparticles in Dense Polymer Melts; J. Phys. Chem. B, 2014, 118, 3731-3742.

(28) Jain, S.; Goossens, J. G. P.; Peters, G. W. M.; van Duin, M.; Lemstra, P. J., Strong Decrease in Viscosity of Nanoparticle-filled Polymer Melts through Selective Adsorption, Soft Matter, 2008, 4, 1848-1854.

(29) Roscoe, R. The Viscosity of Suspensions of Rigid Spheres. Br. J. Appl. Phys. 1952, 3, 267269.

(30) Batchelor, G. K. The Effect of Brownian Motion on the Bulk Stress in a Suspension of Spherical Particles. J. Fluid Mech. 1977, 83, 97-117.

(31) Kholodenko, A. L.; Douglas, J. F. Generalized Stokes-Einstein Equation for Spherical Particle Suspensions. Phys. Rev. E 1995, 51, 1081-1090.

(32) Toda, K.; Furuse, H. Extension of Einstein's Viscosity Equation to that for Concentrated Dispersions of Solutes and Particles. J. Biosci. Bioeng. 2006, 102, 524-528. 
(33) Burgos-Mármol, J. J.; Patti, A.; Unveiling the Impact of Nanoparticle Size Dispersity on the Behavior of Polymer Nanocomposites, Polymer, 2017, 113, 92-104.

(34) Tae Jung, P.; Sung, B. J.; Yethiraj, A.; The Influence of the Polymer Chain Stiffness on Tracer Diffusion in Polymeric Matrices; J. Polym. Sci. Part B: Polym. Phys., 2011, 49, 818-825.

(35) Yethiraj, A.; Fynewever, H. Isotropic to Nematic Transition in Semiflexible Polymer Melts, Mol. Phys. 1998, 93:5, 693-701.

(36) McGrother, S. C.; Williamson, D. C.; Jackson, G. A Reexamination of the Phase Diagram of Hard Spherocylinders, J. Chem. Phys. 1996, 104, 6755-6771.

(37) Bolhuis, P.; Frenkel, D. Tracing the Phase Boundaries of Hard Spherocylinders, J. Chem. Phys. 1997, 106, 666-687.

(38) Cuetos, A.; Martínez-Haya, B.; Lago, S. Liquid Crystal Behavior of the Kihara fluid, Phys. Rev. E 2003, 68, 011704.

(39) Schmidt, M.; Dijkstra, M. Isotropic-Nematic Transition of Hard Rods Immersed in Random Sphere Matrices, J. Chem. Phys. 2004, 121, 12067-12073.

(40) Orlandi, S.; Benini, E.; Miglioli, I.; Evans, D. R.; Reshetnyak, V.; Zannoni, C. Doping Liquid Crystals with Nanoparticles. A Computer Simulation of the Effects of Nanoparticle Shape, Phys. Chem. Chem. Phys. 2016, 18, 2428.

(41) Yuan, Q. W.; Mark, J. E. Reinforcement of Poly(dimethylsiloxane) Networks by Blended and In-situ Generated Silica Fillers Having Various Sizes, Size distributions, and Modified Surfaces, Macromol. Chem. Phys. 1999, 200, 206-220.

(42) Plimpton, S. Fast Parallel Algorithms for Short-Range Molecular Dynamics, J. Comp. Phys., 1995, 117, 1-19.

(43) Liu, J.; Cao, D.; Zhang, L. Molecular Dynamics Study on Nanoparticle Diffusion in Polymer Melts: A Test of the Stokes-Einstein Law, J. Phys. Chem. C 2008, 112, 6653-6661. 
(44) Hattemer, G.; Arya, G. Viscoelastic Properties of Polymer-Grafted Nanoparticle Composites from Molecular Dynamics Simulations, Macromololecules 2015, 48, 1240-1255.

(45) Hagita, K.; Morita, H.; Doi, M.; Takano, H. Coarse-Grained Molecular Dynamics Simulation of Filled Polymer Nanocomposites under Uniaxial Elongation Macromololecules 2015, 49, 1972-1983.

(46) Zhou, X.; Jiang, Y.; Deng, Z.; Zhang, L. Glassy Dynamics of Nanoparticles in Semiflexible Ring Polymer Nanocomposite Melts, Sci. Rep. 2017, 7, 44325.

(47) Reith, D.; Meyer, H.; Müller-Plathe, F. Mapping Atomistic to Coarse-Grained Polymer Models Using Automatic Simplex Optimization to Fit Structural Properties, Macromolecules 2001, 34, 2335-2345.

(48) Fukunaga, H.; Aoyagi, T.; Takimoto, J.-i.; Doi, M. Derivation of Coarse-Grained Potential for Polyethylene, Comp. Phys. Commun. 2001, 142, 224-226.

(49) Chen, L.I.; Qian, H.-J.; Lu, Z.-Y.; Li, Z.-S.; Sun, C.-C. An Automatic Coarse-Graining and Fine-Graining Simulation Method: Application on Polyethylene, J. Phys. Chem. B 2006, 110, 24093-24100.

(50) Curcó, D.; Alemán, C. Coarse-Grained Simulations of Amorphous and Melted Polyethylene, Chem. Phys. Lett. 2007, 436, 189-193.

(51) Panizon, E.; Bochicchio, D. Monticelli, L.; Rossi, G. MARTINI Coarse-Grained Models of Polyethylene and Polypropylene, J. Phys. Chem. B 2015, 119, 8209-8216.

(52) Carbone, P.; Varzaneh, H. A. K.; Chen, X.; Müller-Plathe, F. Transferability of CoarseGrained Force Fields: the Polymer Case, J. Chem. Phys. 2008, 128, 064904.

(53) Groot, R. D.; Warren, P. B. Dissipative Particle Dynamics: Bridging the Gap between Atomistic and Mesoscopic Simulation, J. Chem. Phys., 1997, 107, 4423. 
(54) Español, P.; Warren, P. B. Statistical Mechanics of Dissipative Particle Dynamics, Europhys. Lett., 1995, 30, 191.

(55) Bonet-Ávalos, J.; Mackie, A. D. Dissipative Particle Dynamics with Energy Conservation, Europhys. Lett., 1997, 40, 141-146.

(56) Liu, J.; Cao, D.; Zhang, L. Molecular Dynamics Study on Nanoparticle Diffusion in Polymer Melts: A Test of the Stokes-Einstein Law. J. Phys. Chem. C 2008, 112, 6653-6661.

(57) Müller-Plathe, F. Reversing the Perturbation in Nonequilibrium Molecular Dynamics: the Shear Viscosity of Fluids, Phys. Rev. E. 1999, 59, 4894-4898.

(58) Müller-Plathe, F.; Bordat, P. Reverse Non-Equilibrium Molecular Dynamics, Lect. Notes Phys. 2004, 640, 310-326.

(59) Löwen, H. Anisotropic Self-Diffusion in Colloidal Nematic Phases, Phys. Rev. E 1999, 59, 1989-1995.

(60) Chen, X.; Carbone, P.; Cavalcanti, W. L.; Milano, G.; Müller-Plathe, F. Viscosity and Structural Alteration of a Coarse-Grained Model of Polystyrene under Steady Shear Flow Studied by Reverse Nonequilibrium Molecular Dynamics, Macromolecules 2007, 40, 8087-8095.

(61) Miesowicz, M. The Three Coefficients of Viscosity of Anisotropic Liquids, Nature 1946, $158,27-27$.

(62) Kratky, O.; Porod, G. Röntgenuntersuchung gelöster Fadenmoleküle, Rec. Trav. Chim. 1949, $68,1106$.

(63) Hsu, H.-P.; Paul, W.; Binder, K. Standard Definitions of Persistence Length Do Not Describe the Local "Intrinsic" Stiffness of Real Polymer Chains, Macromolecules 2010, 43, 30943102. 
(64) Egorov, S. A.; Milchev, A.; Virnau, P.; Binder, K. A New Insight into the IsotropicNematic Phase Transition in Lyotropic Solutions of Semiflexible Polymers: Density-Functional Theory Tested by Molecular Dynamics, Soft Matter 2016, 12, 4944-4959.

(65) Chen, Z. Y. Nematic Ordering in Semiflexible Polymer Chains, Macromolecules 1993, 26, 3419-3423.

(66) Dogic, Z.; Fraden, S. Phase Behavior of Rod-like Viruses and Virus/Sphere Mixtures, Soft Matter, Volume 2: Complex Colloidal Suspensions, (eds G. Gompper and M. Schick), WileyVCH Verlag GmbH, Weinheim, 2006, Chapter 1.

(67) Naderi, S.; van der Schoot, P. Effect of Bending Flexibility on the Phase Behavior and Dynamics of Rods, J. Chem. Phys. 2014, 141, 124901.

(68) Cuetos, A.; Patti, A. Equivalence of Brownian dynamics and Dynamic Monte Carlo Simulations in Multicomponent Colloidal Suspensions, Phys. Rev. E 2015, 92, 022302.

(69) Lettinga, M. P.; Barry, E.; Dogic, Z. Self-diffusion of Rod-Like Viruses in the Nematic Phase, Europhys. Lett. 2005, 71, 692-698.

(70) Park, C. H.; Sung, B. J. Dynamics of Nano-Particles in Polymer Nanocomposites: A Molecular DynamicsSimulation Study, J. Chem. Biol. Interfaces 2013, 1, 35-40.

(71) Daivis, P. J.; Matin, M. L.; Todd, B. D. Nonlinear Shear and Elongational Rheology of Model Polymer Melts by Non-Equilibrium Molecular Dynamics, J. Non-Newtonian Fluid Mech. 2003, 111, 1-18.

(72) Xu, Z.; de Pablo, J. J.; Kim, S. Transport Properties of Polymer Melts from Nonequilibrium Molecular Dynamics, J. Chem. Phys. 1995, 102, 5836-5844.

(73) Bosko, J. T.; Todd, B. D.; Sadus, R. J. Viscoelastic Properties of Dendrimers in the Melt from Nonequlibrium Molecular Dynamics, J. Chem. Phys. 2004, 121, 12050-12059. 
(74) Doi, M.; Ewards, S. F. The Theory of Polymer Dynamics, Oxford University Press, New York, 1986.

(75) Müller, M.; Wittmer, J. P.; Barrat, J.-L. On Two Intrinsic Length Scales in Polymer Physics: Topological Constraints vs. Entanglement Length, Europhys. Lett. 2000, 52, 406-412. 


\section{Graphical TOC Entry}

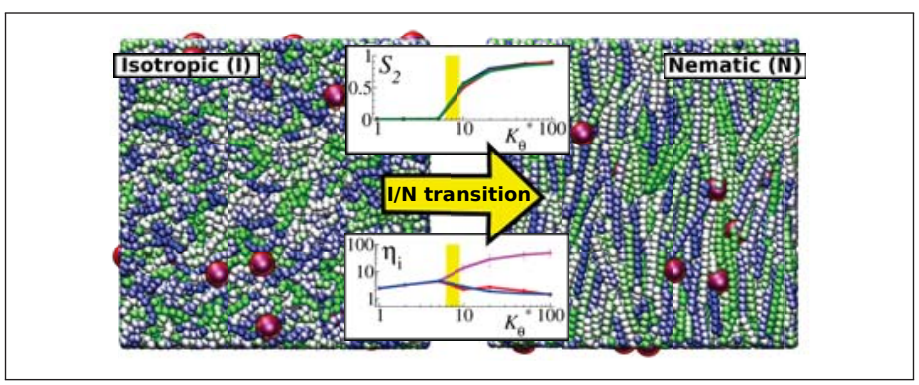

